\title{
INHIBITION-BASED RELAXATION OSCILLATIONS EMERGE IN RESONATOR NETWORKS
}

\author{
Andrea Bel ${ }^{1,2, *}$, Ana Torresi ${ }^{1}$ And Horacio G. Rotstein Rot, $^{3, *}$
}

\begin{abstract}
We investigate the mechanisms responsible for the generation of oscillations in mutually inhibitory cells of non-oscillatory neurons and the transitions from non-relaxation (sinusoidal-like) oscillations to relaxation oscillations. We use a minimal model consisting of a $2 \mathrm{D}$ linear resonator, a 1D linear cell and graded synaptic inhibition described by a piecewise linear sigmoidal function. Individually, resonators exhibit a peak in their response to oscillatory inputs at a preferred (resonant) frequency, but they do not show intrinsic (damped) oscillations in response to constant perturbations. We show that network oscillations emerge in this model for appropriate balance of the model parameters, particularly the connectivity strength and the steepness of the connectivity function. For fixed values of the latter, there is a transition from sinusoidal-like to relaxation oscillations as the connectivity strength increases. Similarly, for fixed connectivity strength values, increasing the connectivity steepness also leads to relaxation oscillations. Interestingly, relaxation oscillations are not observed when the 2D linear node is a damped oscillator. We discuss the role of the intrinsic properties of the participating nodes by focusing on the effect that the resonator's resonant frequency has on the network frequency and amplitude.
\end{abstract}

Mathematics Subject Classification. 92C20, 37C27, 37N25.

Received November 4, 2018. Accepted April 23, 2019.

\section{INTRODUCTION}

Oscillatory patterns are ubiquitous in chemical, biochemical and biological systems $[9,23,25,41,57,75,82$, 95]. The dynamic mechanisms of generation of oscillations require the interplay of positive (relatively fast) and negative (relatively slow) feedback effects. In chemical and biochemical oscillators [5, 29, 41, 99], the so-called activators favor both changes in its own production via autocatalytic effects and the production of the so-called inhibitors. The latter oppose changes in the activator on a slower time scale that allows the activator to reach

Keywords and phrases: Neural networks, resonance, relaxation oscillation, canard phenomenon.

1 Departamento de Matemática, Universidad Nacional del Sur, 8000 Bahía Blanca, Argentina.

2 INMABB, CONICET, Bahía Blanca, Argentina.

${ }^{3}$ Federated Department of Biological Sciences, New Jersey Institute of Technology and Rutgers University, Newark, NJ, USA.

${ }^{4}$ Institute for Brain and Neuroscience Research, New Jersey Institute of Technology, Graduate Faculty, Behavioral Neurosciences Program, Rutgers University, Newark, NJ, USA.

* Corresponding author: andrea.bel@uns.edu.ar

** HGR is a member of the graduate faculty in the Behavioral Neurosciences Program at Rutgers University and a Corresponding Investigator at CONICET, Argentina. 
high enough levels. In neurons, the necessary negative and positive feedback effects are provided by the so-called resonant and amplifying gating variables, respectively, associated to neuronal ionic currents [40, 66, 72, 74].

The properties of these oscillatory patterns in single oscillators depend on the relative levels of the positive and negative feedback effects, which are controlled by the systems' nonlinearities and time scales [30, 82]. These, in turn, depend on the physical properties of the specific systems under study. Linear systems can exhibit "at most" damped oscillations, but not sustained ones. The emergence of sustained (limit cycle) oscillations require the presence of nonlinearities and high enough levels of the amplifying process. As the time scale separation between the activator (e.g. neuronal voltage) and the negative feedback variable (e.g. ionic resonant gating variable, inhibitor, repressor) increases, the oscillations transition from sinusoidal-like to relaxation oscillations.

A phenomenon closely related to the presence of oscillations is that of resonance in single cells, defined as their ability to exhibit a peak in the amplitude response of their main variable (e.g. voltage) to oscillatory inputs at a preferred (resonant) input frequency [40,66, 70, 74]. This phenomenon has been distinctly observed both experimentally and theoretically in the membrane potential of neurons operating at subthreshold levels and it has been termed membrane potential resonance (MPR) or subthreshold resonance $[2,3,7,8,12,15,16,22,24$, 27, 28, 32-35, 37-40, 44-46, 49, 53, 56, 58-71, 74, 76, 77, 80, 81, 83, 86, 90, 91, 96-98]. Dynamical systems, even two-dimensional linear systems with real eigenvalues can exhibit resonance [66, 74]. We refer to the neurons, and in general to the dynamical systems, that can exhibit resonance in the absence of intrinsic oscillations (damped or sustained) as resonators. Typically, resonators exhibit an overshoot response to constant inputs. Both this overshoot and resonance are generated by the interplay of the same types feedback effects as intrinsic oscillations, but in a different regime, with lower amplifying process levels [66, 69, 70].

In addition to the single-cell mechanisms of generation of oscillations described above, oscillations can be generated at the network level in mutually connected cells that do not exhibit sustained oscillations when disconnected, but they are rather damped oscillators or resonators $[4,48,88,93]$. The graded synaptic connectivity used in these studies has been assumed to be instantaneously fast and to have no dynamics. Graded synapses are present in several neuronal systems $[1,10,14,17,47,48,78,84,93]$.

In [4] we have identified a minimal (3D) network model consisting of a two-dimensional resonator and a one-dimensional passive cell mutually connected through graded inhibition. Oscillations are also obtained in self-excited resonators (2D), mutually excited resonators (4D) and mutually inhibited resonators (4D), also using graded synaptic connectivity. In self-excited networks, self-excitation provides the amplifying process necessary for the generation of oscillations. In fact, self-excited resonators have the same mathematical structure as single neurons having both resonant and amplifying ionic processes whose dynamics are also 2D [4]. Mutually excited resonators exhibit synchronized in-phase patterns and therefore their dynamics are captured by those of self-excited resonators. In mutually inhibited two-cell networks involving at least one resonator (e.g. resonator/resonator or resonator/passive cell networks), in contrast, the amplifying process is more complex and involves the combined activity of the two inhibitory synaptic connectivities through the intrinsic properties of the nodes.

The goal of this paper is to identify the conditions under which mutually inhibitory resonator/passive cell networks exhibit relaxation oscillations and to understand the mechanisms that govern the transition from sinusoidal-like to relaxation oscillations. To this end we use a minimal network model consisting of (i) a 2D linear resonator, (ii) a 1D linear passive cell, and (iii) graded synaptic connectivity. We primarily used graded synapses of sigmoidal piecewise linear (PWL) type. A secondary goal of this paper is to identify the differences between the oscillatory patterns produced in models with PWL and smooth synaptic connectivity.

The overview of the paper is as follows. In Section 2, we describe the mathematical network model and the mathematical tools we use in our study. In Section 3, we present our results. First, we discuss the basic aspects of the network dynamic oscillatory structure, including the description of the fixed-points, the bifurcations that give rise to oscillations for low values of the maximal synaptic conductance (connectivity strength) $\left(G_{i n}\right)$, the transition from sinusoidal to relaxation oscillations as $G_{i n}$ increases, and the abrupt termination of the oscillations in a non-smooth bifurcation for higher values of $G_{i n}$. Then, we describe the necessary balances between the two parameters capturing the synaptic connectivity, $G_{i n}$ and the synaptic connectivity gradedness (captured by a parameter $v_{a}$ ), for the network oscillations to exist. For fixed-values of $G_{i n}$, relaxation oscillations 
require the connectivity function to be steep enough. Subsequently, we show how the resonant frequency of the individual 2D resonator affects the network oscillation frequency. Finally, we review some results obtained in [4] for smooth connectivity functions, discuss the existence of relaxation oscillations and the associated canard phenomenon, and compare the oscillatory properties of networks having smooth and PWL connectivity functions. We conclude in Section 4 by discussing our results, their implications for network dynamics and future work.

\section{Model And mathematical tools}

We consider a minimal network model consisting of two mutually inhibitory cells (Fig. 1A). The dynamics of the individual cells are described by linearized biophysical (conductance-based) models [66, 74]. One of the cells is a two-dimensional (2D) resonator (blue) and the other one is a one-dimensional (1D) passive cell (red). Both are linearized around a fixed-point in the subthreshold regime. The network connectivity is implemented using graded synapses, active at subthreshold levels.

The network model is described by the following equations

$$
\begin{aligned}
C_{1} \frac{\mathrm{d} v_{1}}{\mathrm{~d} t} & =-g_{L, 1} v_{1}-g_{1} w_{1}-G_{i n, 21} S_{\infty}\left(v_{2}\right)\left(v_{1}-E_{i n}\right), \\
\tau_{1} \frac{\mathrm{d} w_{1}}{\mathrm{~d} t} & =v_{1}-w_{1}, \\
C_{2} \frac{\mathrm{d} v_{2}}{\mathrm{~d} t} & =-g_{L, 2} v_{2}-G_{i n, 12} S_{\infty}\left(v_{1}\right)\left(v_{2}-E_{i n}\right),
\end{aligned}
$$

where $v_{1}$ and $v_{2}$ represent the voltage $(\mathrm{mV}), w_{1}$ represents the normalized gating variable for the resonant ionic current, $t$ is time (ms), $C_{1}$ and $C_{2}$ are the membrane capacitances $\left(\mu \mathrm{F} / \mathrm{cm}^{2}\right), g_{L, 1}$ and $g_{L, 2}$ are the linearized leak maximal conductances $\left(\mathrm{mS} / \mathrm{cm}^{2}\right), g_{1}$ is the ionic current linearized conductance $\left(\mathrm{mS} / \mathrm{cm}^{2}\right)$ and $\tau_{1}$ is the linearized time constant $(\mathrm{ms})$. The last term in the first and third equations represents the inhibitory graded synaptic current from the other neuron, $G_{i n, j k}$ is the maximal conductance, $E_{i n}$ is the synaptic reversal potential (referred to the resting potential) and the connectivity function $S_{\infty}$ is a function of sigmoid type.

We consider two different functions $S_{\infty}$, the sigmoid function

$$
S_{\infty}(v)=\left(1+e^{-\frac{v-v_{h l f}}{v_{s l p}}}\right)^{-1}
$$

where $v_{h l f}$ is the half-activation point and $v_{s l p}$ is a positive constant (Fig. 1B, gray), and a piecewise-linear (PWL) function of sigmoid type given by

$$
S_{\infty}(v)= \begin{cases}0 & \text { if } v \leq v_{b} \\ \left(v_{a}-v_{b}\right)^{-1}\left(v-v_{b}\right) & \text { if } \quad v_{b}<v<v_{a} \\ 1 & \text { if } v \geq v_{a}\end{cases}
$$

where $v_{a}$ and $v_{b}$ are real constants (Fig. 1B, blue).

We consider graded synapses that operate at the subthreshold voltage level regime. These synapses can be partially activated when the voltage is close to a certain threshold value (not related to the voltage threshold for spike generation, which is outside the scope of this paper). In our model the values of $v_{s l p}, v_{a}$ and $v_{b}$ determine the gradedness of activation for the functions (2.2) and (2.3), respectively. In the following sections we consider connections centered at $v=0\left(v_{h l f}=0\right.$ and $\left.v_{b}=-v_{a}\right)$, and values of $v_{s l p}$ and $v_{a}$ such that the connections act in a range of, at most, $10 \mathrm{mV}$ (see Fig. 1B). 
A

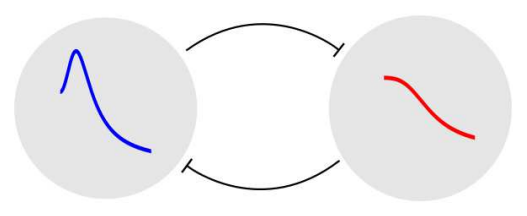

B

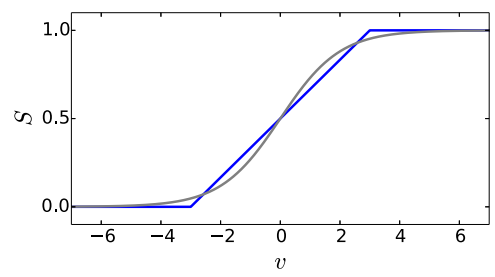

Figure 1. (A) Network diagram for the mutually inhibitory resonator (blue) - passive cell (red) model. (B) Connectivity functions $S_{\infty}(v)$ : PWL sigmoid function (2.3) with $v_{b}=-3$ and $v_{a}=3$ (blue) and smooth sigmoid function (2.2) with $v_{h l f}=0$ and $v_{s l p}=1$ (gray).

We note that $v_{i}$ in (2.1) is the voltage relative to the coordinate of the equilibrium potential $\bar{V}_{i}$ for the conductance-based model (A.1)-(A.3) (see in Appendix A.1 the description of this model). In addition, $E_{i n}$ in (2.1) is the synaptic reversal potential relative to $\bar{V}_{i}$. Unless stated otherwise, we used the parameter values $C_{1}=C_{2}=1$ and $E_{i n}=-20$.

\subsection{Fixed-point stability and Andronov-Hopf bifurcation}

We calculate the stability of the fixed-points of system (2.1) considering the linearization and the corresponding eigenvalues. It is important to mention that the network with the PWL connection given in (2.3) is a non-smooth system. Thus, the state space is divided in different region by the transition planes $v_{1}=v_{b}, v_{1}=v_{a}$, $v_{2}=v_{b}$ and $v_{2}=v_{a}$. In each region the system has, at most, quadratic non-linearities, and the model could be linearized to determine the stability of the fixed-points belonging to that region. If a fixed-point belongs to one of the transition planes it is called a transition fixed-point [19].

To study the local bifurcation of periodic orbits we apply a version of the Hopf bifurcation theorem in the frequency domain $[52,55]$. This approach allows us to determine regions of the parameter space where periodic solutions exist and to describe their characteristics in a small neighbourhood of the bifurcation point. Here we briefly show how the frequency domain method is applied to our model. A more general description is presented in the Appendix A.2.

The network model (2.1) can we written as follows

$$
\left(\begin{array}{c}
\dot{v_{1}} \\
\dot{v_{2}} \\
\dot{w_{1}}
\end{array}\right)=A\left(\begin{array}{c}
v_{1} \\
v_{2} \\
w_{1}
\end{array}\right)+B u
$$

where

$$
\begin{aligned}
& u=-h\left(C \cdot\left(v_{1}, v_{2}, w_{1}\right)^{T}, G_{i n}\right)=-h\left(v_{1}, v_{2}, G_{i n}\right)=\left(\begin{array}{c}
G_{i n}\left(v_{1}-E_{i n}\right) S_{\infty}\left(v_{2}\right) \\
G_{i n}\left(v_{2}-E_{i n}\right) S_{\infty}\left(v_{1}\right)
\end{array}\right), \\
& A=\left(\begin{array}{ccc}
-g_{L, 1} & 0 & -g_{1} \\
0 & -g_{L, 2} & 0 \\
\epsilon & 0 & -\epsilon
\end{array}\right), \quad B=\left(\begin{array}{ll}
1 & 0 \\
0 & 1 \\
0 & 0
\end{array}\right)^{T}, \quad C=\left(\begin{array}{lll}
1 & 0 & 0 \\
0 & 1 & 0
\end{array}\right),
\end{aligned}
$$

and $\epsilon=1 / \tau$.

The above is an input-output representation of the model, where the feedback effect is provided by the function $u=-h\left(v_{1}, v_{2}, G_{i n}\right), u$ is the vector of inputs, $\left(v_{1}, v_{2}\right)$ is the vector of outputs and $G_{i n}$ is the 
main bifurcation parameter. By using this representation, the system is described through a transfer function $\mathcal{G}(s) \in \mathbb{C}^{2 \times 2}$, given by

$$
\mathcal{G}(s)=C\left(s I_{3}-A\right)^{-1} B=\left(\begin{array}{cc}
\frac{s+\epsilon}{s^{2}+s\left(g_{L, 1}+\epsilon\right)+\epsilon\left(g_{l}+g_{L, 1}\right)} & 0 \\
0 & \frac{1}{s+g_{L, 2}}
\end{array}\right),
$$

where $s$ is the Laplace variable and $I_{3}$ is the $3 \times 3$ identity matrix.

Let $\left(\hat{v}_{1}, \hat{v}_{2}\right)$ an equilibrium, calculated as a solution of $\mathcal{G}(0) h\left(v_{1}, v_{2}, G_{i n}\right)+\left(v_{1}, v_{2}\right)^{T}=0$. Then, by linearizing around this equilibrium we obtain the gain matrix

$$
J=\left.\frac{\partial h}{\partial\left(v_{1}, v_{2}\right)}\right|_{\left(\hat{v}_{1}, \hat{v}_{2}\right)}=\left(\begin{array}{cc}
-G_{i n} S_{\infty}\left(\hat{v}_{2}\right) & -G_{i n}\left(\hat{v}_{1}-E_{i n}\right) S_{\infty}^{\prime}\left(\hat{v}_{2}\right) \\
-G_{i n}\left(\hat{v}_{2}-E_{i n}\right) S_{\infty}^{\prime}\left(\hat{v}_{1}\right) & -G_{i n} S_{\infty}\left(\hat{v}_{1}\right)
\end{array}\right),
$$

and an open-loop transfer function $\mathcal{G}(s) J$ for which the polynomial $\operatorname{det}\left(\lambda I_{2}-\mathcal{G}(s) J\right)=0$, where $I_{2}$ the $2 \times 2$ identity matrix, defines $\lambda_{k}\left(s, G_{i n}\right)$ characteristic functions, $k=1,2$.

It has been proven $[52,55]$ that in an Andronov-Hopf bifurcation point the open-loop transfer matrix $\mathcal{G}(i \omega) J$ has a distinguished simple characteristic function, noted as $\hat{\lambda}\left(\omega, G_{i n}\right)$, such that for a unique frequency $\omega_{0}$ and a critical value of the parameter $G_{i n 0}$, satisfies $\hat{\lambda}\left(\omega_{0}, G_{i n 0}\right)=-1$ and the graphic of $\hat{\lambda}\left(\omega, G_{i n}\right)$ crosses the critical value -1 in the complex plane when the bifurcation parameter varies.

Once a frequency $\omega_{0}$ and a critical value $G_{i n 0}$ are determined, by using the Hopf bifurcation theorem in frequency domain (see Appendix A.2), we obtain a scalar equation in $\mathbb{C}$ of the form

$$
\theta\left(\hat{\lambda}\left(\omega, G_{\text {in }}\right)+1+\theta^{2} \xi_{1}\left(\omega, G_{\text {in }}\right)+\mathcal{O}\left(\theta^{2}\right)\right)=0
$$

whose non-zero solutions are in one-to-one correspondence with the periodic small amplitude solutions of system (2.4). The constant $\theta$ is an amplitude measure and the solutions have period close to $2 \pi / \omega_{0}$.

From the above equation, for values of the amplitude $\theta \neq 0$ sufficiently small, we obtain the following equations in $\mathbb{R}$

$$
G_{i n}=G_{i n 0}+G_{i n 2} \theta^{2}+\cdots,
$$

and

$$
\omega=\omega_{0}+\omega_{2} \theta^{2}+\cdots
$$

The first equation is the expression of the bifurcation of periodic solutions and it relates the main bifurcation parameter $G_{i n}$ and the amplitude $\theta$, characterizing the Hopf bifurcation phenomenon. The second expression gives the modification of the frequency in terms of the variation of the amplitude.

Moreover, we obtain an approximated expression for the small amplitude solutions near the critical value of the parameter, up to order two the approximation results

$$
\left(v_{1}(t), v_{2}(t)\right)=\left(\hat{v_{1}}, \hat{v_{2}}\right)+e_{2}\left(\omega, G_{i n}\right) \exp (i 2 \omega t)+\mathcal{O}\left(\theta^{2}\right) .
$$

where the vector $e_{2}\left(\omega, G_{i n}\right) \in \mathbb{R}^{2}$ is obtained with the iterative method, and $G_{i n}$ and $\omega$ are defined in (2.6) and (2.7), respectively.

In the following section we use the expressions (2.6), (2.7) and (2.8), to find the Andronov-Hopf bifurcation points and to describe the branch of periodic solutions of (2.1) generated in these bifurcations. In addition, the expressions of $G_{i n 0}$ and $G_{i n 2}$ in (2.6), allows us to perform a general exploration of the parameter space in 
order to study the existence of small amplitude periodic solutions when an auxiliary parameter $\left(e . g . g_{L, 1}, g_{L, 2}\right.$, $v_{s l p}$ or $v_{a}$ ) is varying.

\subsection{Slow-fast system}

In this subsection we rewrite the model (2.1) as a fast-slow system to help in the determination of possible canard explosions. In planar systems this type of studies is based in Fenichel results and their generalizations $[6,21,26,42,50,51]$. Our model is three dimensional with one slow variable and two fast variables, and there are not general results to study these cases [43]. However, we use the critical manifold, its projections and the inflexion points near it, to predict the existence of possible canard explosions (see Sect. 3.8). This analysis is then corroborated by numerical simulations. We perform a preliminary study of the canard explosions because the global dynamic of the three dimensional one slow two fast systems is difficult to reconstruct from the singular limit fast-slow decomposition.

For large enough values of $\tau_{1}$, system (2.1) has two time scales. We substitute $\epsilon=1 / \tau_{1}$ in model (2.1) with the connection (2.2), for $0<\epsilon \ll 1$. We refer to the resulting system

$$
\begin{aligned}
v_{1}^{\prime} & =f_{1}\left(v_{1}, v_{2}, w_{1}, G_{i n}\right), \\
v_{2}^{\prime} & =f_{2}\left(v_{1}, v_{2}, G_{i n}\right), \\
w_{1}^{\prime} & =\epsilon\left(v_{1}-w_{1}\right)=f_{3}\left(v_{1}, w_{1}\right),
\end{aligned}
$$

as $(2,1)$-fast-slow, where $v_{1}$ and $v_{2}$ are the fast variables, $w_{1}$ is the slow variable and $(\cdot)^{\prime}=\frac{\mathrm{d}}{\mathrm{d} t}$. The functions $f_{1}$ and $f_{2}$ are the right-hand-sides in the first and third eqs. in (2.1). There is an associated slow time $t_{s}$ such that $t_{s}=\epsilon t$.

The critical manifold for the system is the solution of $f_{1}\left(v_{1}, v_{2}, w_{1}, G_{i n}\right)=0$ and $f_{2}\left(v_{1}, v_{2}, G_{i n}\right)=0$. Because of the linear terms in the above equations, for fixed values of the maximal conductance $G_{i n}$, we obtain the following critical curve

$$
C=C\left(G_{\text {in }}\right)=\left\{\left(v_{1}, v_{2}, w_{1}\right) \in \mathbb{R}^{3}: v_{1} \in \mathbb{R}, v_{2}=p_{2}\left(v_{1}, G_{\text {in }}\right), w_{1}=p_{1}\left(v_{1}, G_{\text {in }}\right)\right\},
$$

where

$$
p_{2}\left(v_{1}, G_{i n}\right)=\frac{G_{i n, 12} S_{\infty}\left(v_{1}\right) E_{i n}}{g_{L, 2}+G_{i n, 12} S_{\infty}\left(v_{1}\right)} \quad p_{1}\left(v_{1}, G_{i n}\right)=-\frac{1}{g_{1}}\left(g_{L, 1} v_{1}+G_{i n, 21} S_{\infty}\left(p_{2}\left(v_{1}, G_{i n}\right)\right)\left(v_{1}-E_{i n}\right)\right) .
$$

Since we have two bases time scales to formulate the equations, we get two systems related with (2.9). The fast subsystem results

$$
\begin{aligned}
v_{1}^{\prime} & =f_{1}\left(v_{1}, v_{2}, w_{1}, G_{i n}\right), \\
v_{2}^{\prime} & =f_{2}\left(v_{1}, v_{2}, G_{i n}\right), \\
w_{1}^{\prime} & =0
\end{aligned}
$$

Using Fenichel's results, the information about the fast subsystem and the slow flow are studied to understand the full system $(2.1)[26,43]$.

Each point in $C$ is a fixed point of (2.12). The linearization matrix at $\left(v_{1}, v_{2}\right) \in C$ results

$$
J_{\text {fast }}=\left.\frac{\partial\left(f_{1}, f_{2}\right)}{\partial\left(v_{1}, v_{2}\right)}\right|_{C}=\left(\begin{array}{cc}
-g_{L, 1}-G_{i n} S_{\infty}\left(v_{2}\right) & -G_{i n}\left(v_{1}-E_{i n}\right) S_{\infty}^{\prime}\left(v_{2}\right) \\
-G_{i n}\left(v_{2}-E_{i n}\right) S_{\infty}^{\prime}\left(v_{1}\right) & -g_{L, 2}-G_{i n} S_{\infty}\left(v_{1}\right)
\end{array}\right),
$$

where $v_{2}=p_{2}\left(v_{1}, G_{\text {in }}\right)$. 
A

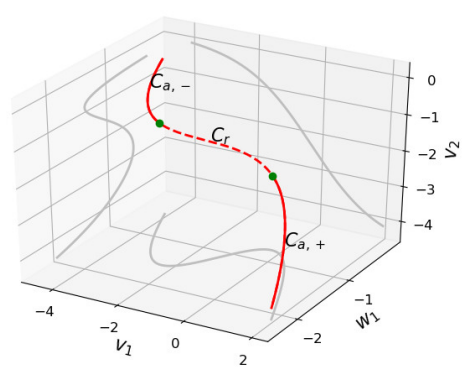

B

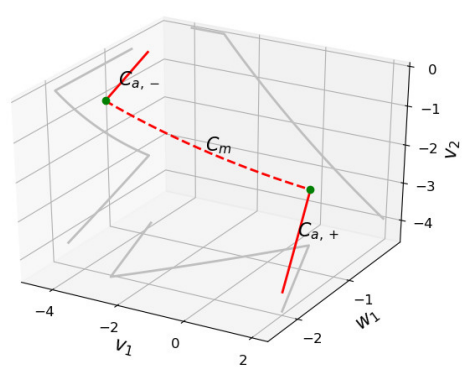

C

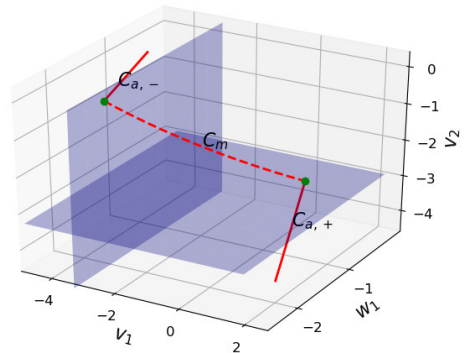

Figure 2. Critical manifold $C$ (red) with its different attracting $\left(C_{a}\right)$ and repelling $\left(C_{r}\right)$ branches, and singular points (dots). Projections of $C$ in two dimensional spaces (gray curves). (A) System with sigmoid function (2.2) where $v_{h l f}=0$ and $v_{s l p}=1$. (B) System with PWL function (2.3) where $v_{b}=-3$ and $v_{a}=3$. (C) System with PWL function (2.3). Critical manifold divided by two transition planes $v_{1}=-3$ and $v_{2}=-3$.

The singular points in $C$ are the points where $J_{\text {fast }}$ is non-invertible. In the present model we have, at most, two singular points that we called $\left(v_{1, \pm}, p_{2}\left(v_{1, \pm}, G_{i n}\right), p_{1}\left(v_{1, \pm}, G_{i n}\right)\right)$, where $\partial p_{1} / \partial v_{1}=0$. The critical manifold is divided in the following attracting $\left(C_{a}\right)$ and repelling $\left(C_{r}\right)$ branches

$$
\begin{aligned}
C_{a,-} & =C \cap\left\{\left(v_{1}, v_{2}, w_{1}\right): v_{1}<v_{1,-}\right\}, \\
C_{r} & =C \cap\left\{\left(v_{1}, v_{2}, w_{1}\right): v_{1,-}<v_{1}<v_{1,+}\right\}, \\
C_{a,+} & =C \cap\left\{\left(v_{1}, v_{2}, w_{1}\right): v_{1}>v_{1,+}\right\} .
\end{aligned}
$$

(Fig. 2A). The attraction or repulsion of the different branches is determined using the linearized matrix (2.13). Each one of the above branches is formed by normally hyperbolic points.

\subsubsection{Slow-fast in the PWL case}

As we already mention, considering the connection $S_{\infty}$ given by (2.3), the system (2.9) is a non-smooth system. We observe that the critical manifold $C$ does not have singular points as we defined before, however, $C$ is divided in different branches by the transition planes (Fig. 2B and C). The stability for each branch is calculated considering the matrix $J_{\text {fast }}$ in the corresponding state space region. Three important branches for our study are

$$
\begin{aligned}
C_{a,-} & =C \cap\left\{\left(v_{1}, v_{2}, w_{1}\right): v_{1}<v_{b}\right\}, \\
C_{m} & =C \cap\left\{\left(v_{1}, v_{2}, w_{1}\right): v_{b}<v_{i}<v_{a}, \quad i=1,2\right\}, \\
C_{a,+} & =C \cap\left\{\left(v_{1}, v_{2}, w_{1}\right): v_{2}<v_{b}\right\} .
\end{aligned}
$$

As in the smooth case considered before, the branches $C_{a}$ are attracting. However, the middle branch $C_{m}$ could be attracting or repelling depending on the value of the maximal connection $G_{i n}$.

Remark 2.1. The fixed-points of the full system (2.1) are the intersection points between the critical curve $C$ and the plane $w_{1}=v_{1}$.

Remark 2.2. We observe that the fast subsystem (2.12) does not present oscillations, that is, the linearization matrix does not have pure imaginary eigenvalues for $S_{\infty}(v)$ of sigmoid type for any fixed point. Therefore, the full system can not present a delayed (dynamic) Hopf bifurcation (see [43]). 


\subsection{Numerical simulations}

The numerical solutions were computed by using a modified Euler method (Runge-Kutta, order 2) [11]. The general time step was $\Delta t=0.01$, and smaller values of $\Delta t$ have been used to improve the accuracy in some limit cases (for example, to calculate homoclinic and heteroclinic orbits). All calculations were performed using Python programming language (Python Software Foundation).

\section{Results}

\subsection{Network model with PWL connectivity function: basic dynamic structure}

Here we consider the model (2.1) with the piecewise-linear (PWL) connectivity function (2.3). We refer to it simply as the PWL model. Note that the PWL model is non-smooth, but not PWL due to the product in the connectivity terms. In the following analysis we consider the maximal conductances $G_{i n, 12}=G_{i n, 21}=G_{i n}$, and $G_{\text {in }}$ as bifurcation parameter.

The fixed-points of the PWL model are the intersection points between the critical manifold $C$ and the plane $w_{1}=v_{1}$. In Figure 3 we show the $\left(v_{1}, w_{1}\right)$-space and the projection of the critical manifold $C$ onto this plane. $C$ is divided in three branches, each one belongs to a different region in the state space (see Fig. 2 right). The transition points belong to $C$ and satisfy

$$
v_{1}=-v_{a} \quad \text { or } \quad v_{1}=-v_{a}-\frac{2 g_{L, 2} v_{a}^{2}}{\left(v_{a}+E_{i n}\right) G_{i n}} .
$$

By increasing the value of $G_{i n}$, the number of fixed-points in the PWL model change. For small values of $G_{i n}$, there exists only one fixed-point located in the middle branch $C_{m}$. For higher values of $G_{i n}$ there are two different cases. If $g_{L, 1}+g_{1} \neq g_{L, 2}$, then first the fixed-point crosses a transition variety and then two more fixedpoints are created in a non-smooth saddle-node bifurcation (Fig. 3A). If $g_{L, 1}+g_{1}=g_{L, 2}$, then a whole segment of non-isolated fixed points is observed in the transition between one and three fixed-points (Fig. 3B). Before other fixed-points appear, by increasing the values of $G_{i n}$, the system displays an Andronov-Hopf bifurcation and the fixed-point in the middle branch of $C$ becomes unstable. We study this bifurcation in detail in the next subsection.

In all cases, the fixed-points in regions $v_{1}<-v_{a}$ or $v_{2}<-v_{a}$ describe the activity of the cells 2 or 1 inhibited, respectively. These fixed-points are stable nodes for all values of the parameters.

\subsection{Andronov-Hopf bifurcation and relaxation oscillations in the PWL model}

Using the frequency domain approach (see Sect. 2.1) we found that the PWL model presents sustained oscillations that are generated in an Andronov-Hopf bifurcation when the maximal conductance $G_{i n}$ is increased above some critical level. The oscillations are generated around the fixed-point in the middle branch of $C$. In these solutions the two nodes oscillate and, because of the mutual inhibition, the synchronization is anti-phase (Figs. 4A and 5A).

If $\tau_{1}$ is large enough, then as $G_{i n}$ increases the amplitude of the limit cycle increases and, eventually, the cycle intersects and cross the transition planes (Fig. 4B and C) visiting up to three regions of the state-space. We can observe that the cycle experiences deformations following the attracting branches of the critical manifold $C$. Thus, if the maximal conductance $G_{i n}$ is increased, a two-time-scale separation develops and the system presents a oscillations of relaxation type (Fig. 4 right column).

For lower values of $\tau_{1}$, we observe sustained oscillations generated in an Andronov-Hopf bifurcation when $G_{i n}$ is increased (Fig. 5). However, these oscillations are not of relaxation type nor relaxation oscillations are observed for other values of $G_{i n}$ because the time scale separation is smaller than in the previous case (Fig. 5 right column). Also, we observe that since the stable cycle remains in two regions of the state-space, near an unstable fixed-point of focus type, the amplitude is smaller than in the previous case. Interestingly, the 2D 
A1

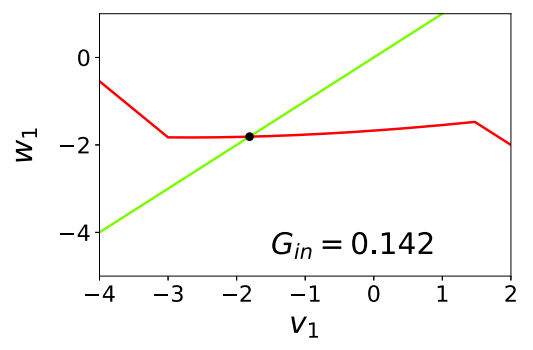

B1

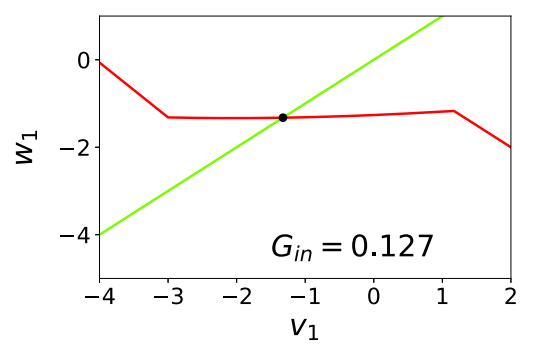

A2

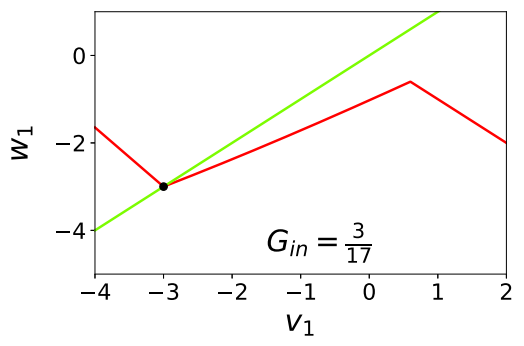

B2

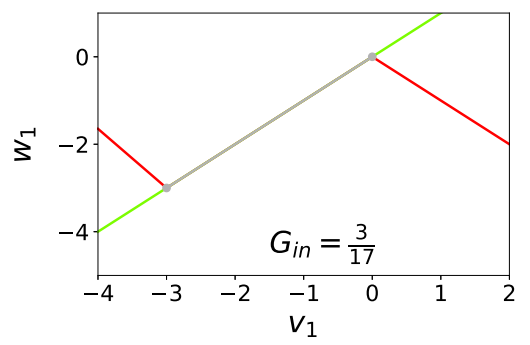

A3

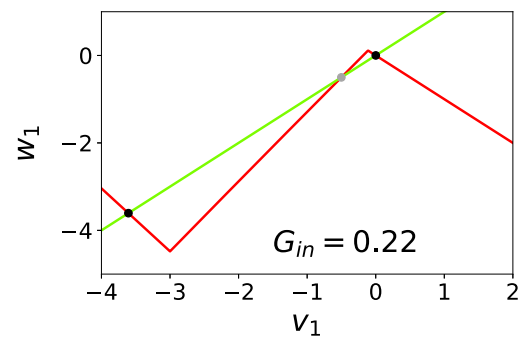

B3

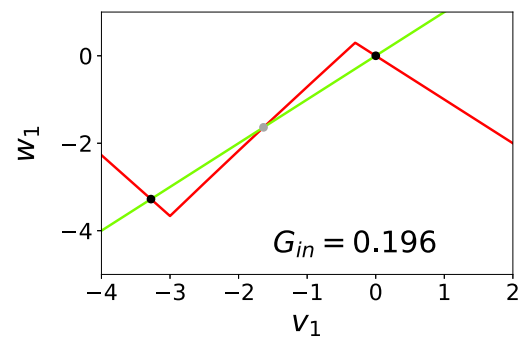

FiguRe 3. Fixed points for the PWL models for representative parameter values. Projections of the critical manifold $C$ (red) and $w_{1}=v_{1}$ (green) in the $\left(v_{1}, w_{1}\right)$-space. Black (gray) dots indicate stable (unstable) fixed-points. (A) $g_{L, 2}=0.6$. (B) $g_{L, 2}=0.5$. We use the following additional parameter values: $g_{L, 1}=0.25, g_{1}=0.25, \tau_{1}=100, v_{a}=3$, and the indicated values of $G_{i n}$.

isolated node 1 shows intrinsic damped oscillations in this case, while it shows resonance, but no intrinsic oscillations for the values of $\tau_{1}$ for which network relaxation oscillations are present.

Figure 6 shows amplitude and frequency of two branches of limit cycles generated in Andronov-Hopf bifurcations. Since the first Lyapunov coefficient $G_{i n 2}$ calculated applying a frequency domain approach is small (see equation (2.6)), after the Andronov-Hopf bifurcation, the amplitude of the limit cycles grows very fast. We can approximate the maximal conductance value when the limit cycle touches a transition plane, in non-smooth systems this is called a grazing bifurcation [20].

As the parameter $G_{i n}$ increases, if $\tau_{1}$ is large enough, the limit cycle cross the different transition planes and its amplitude grows more slowly (Fig. 6A). The frequency of the limit cycle decreases when the parameter is increased and it decreases to zero when $G_{i n}$ tends to $G_{i n, b r}=-2 v_{a}\left(v_{a}+E_{i n}\right)^{-1} \min \left\{g_{L 1}+g_{1}, g_{L 2}\right\}$. The limit cycles disappear abruptly when $G_{i n}=G_{i n, b r}$. In the next subsection we analyze in detail this transition. If the constant time $\tau_{1}$ is smaller, greater values of $G_{i n}$ are necessary to obtain sustained oscillations. The limit cycle shrinks around the fixed-point when $G_{i n}$ increases. In this case, we observe and smooth transition from oscillation to an stable fixed-point.

\subsection{Transition from oscillatory solutions to steady state in the PWL model}

In all cases, the sustained oscillations disappear when the fixed-point in the central branch of the critical manifold $C$ crosses a transition plane as $G_{i n}$ is increased. This behavior is observed when $G_{i n}=G_{i n, b r}=$ $-2 v_{a}\left(v_{a}+E_{i n}\right)^{-1} \min \left\{g_{L 1}+g_{1}, g_{L 2}\right\}$. At that value, the flow in the region $-v_{a}<v_{1,2}<v_{a}$ (the central region) sends trajectories towards the transition planes, and, depending on the rest of the parameters, we observe two cases. If $g_{L, 2} \neq g_{l, 1}+g_{1}$ (see Fig. $3 \mathrm{~A}$ middle), the transition fixed-point $\left(-v_{a},-v_{a}, 0\right)$ attracts the trajectories once they reach the regions $v_{1}<-v_{a}$. Thus, the system has a homoclinic orbit at the (transition) fixed-point (Fig. 7). The homoclinic orbit can reach different regions of the state space and this strongly depends on the 

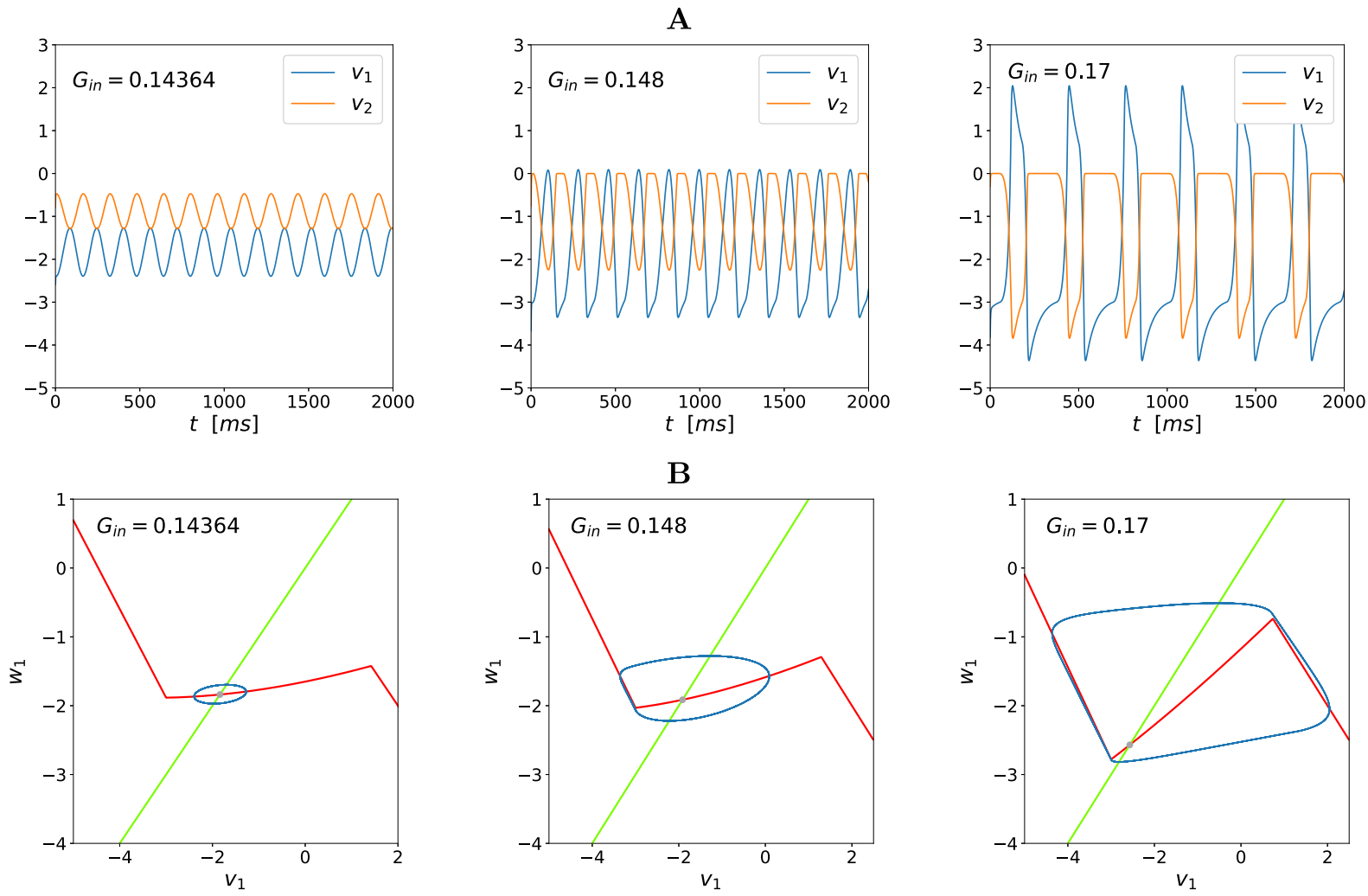

\section{C}
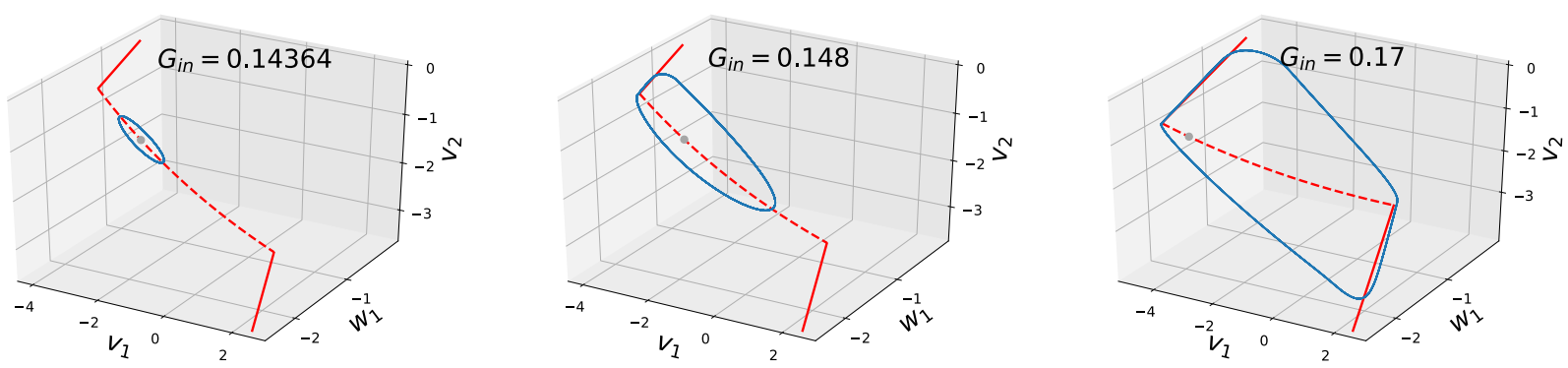

Figure 4. Generation of relaxation oscillations in the PWL model for representative values of $G_{i n}$ and high values of $\tau_{1}$. The values of $G_{i n}$ increase from left to right. (A) Voltage traces (curves of $v$ vs. $t$ ). (B) Cycles in the $\left(v_{1}, w_{1}\right)$-space. (C) Cycles in 3D state-space. We used the following parameter values: $g_{L, 1}=0.25, g_{1}=0.25, g_{L, 2}=0.6, \tau_{1}=100$ and $v_{a}=3$. The critical Andronov-Hopf value is $G_{i n 0}=0.143636$ at the fixed point $v^{*}=$ $(-1.83829,-1.83829,-0.88596)$, and the initial frequency at the Andronov-Hopf bifurcation is $f_{0}=500 \omega_{0} / \pi=6.293384$. Up to order two, the first Lyapunov coefficient results $G_{i n 2}=0.000020458$ indicating the occurrence of a supercritical Andronov-Hopf bifurcation.

time constant values $\tau_{1}$. If $g_{L, 2}=g_{L, 1}+g_{1}$ (see Fig. 3B middle), a segment of non-isolated unstable fixed-points is observed and there are two transition fixed-points, $\left(-v_{a},-v_{a}, 0\right)$ and $\left(0,0,-v_{a}\right)$, attracting the trajectories in the regions $v_{1}<-v_{a}$ and $v_{2}<-v_{a}$, respectively. In this case, we observe that the system has a family 

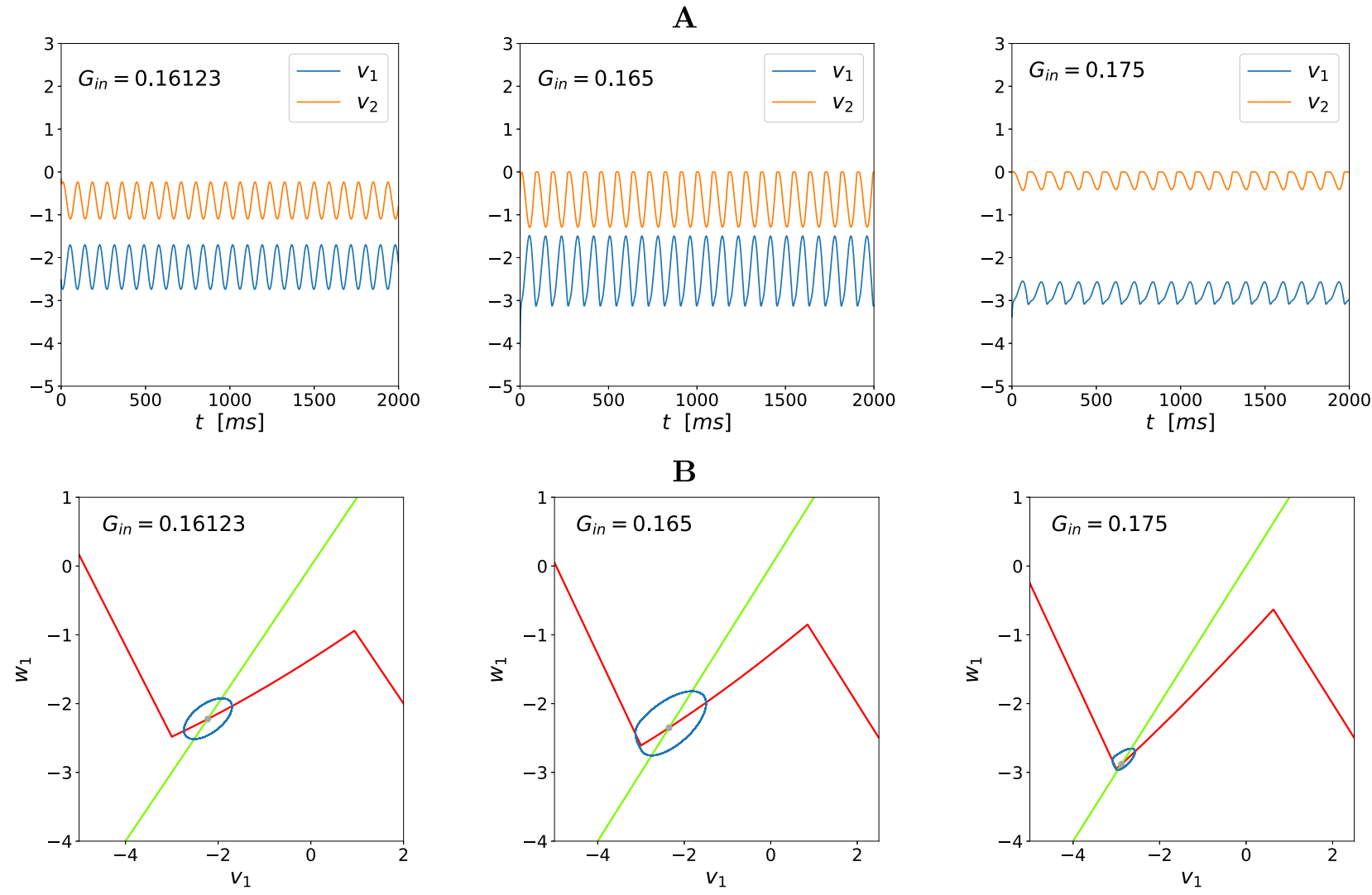

B
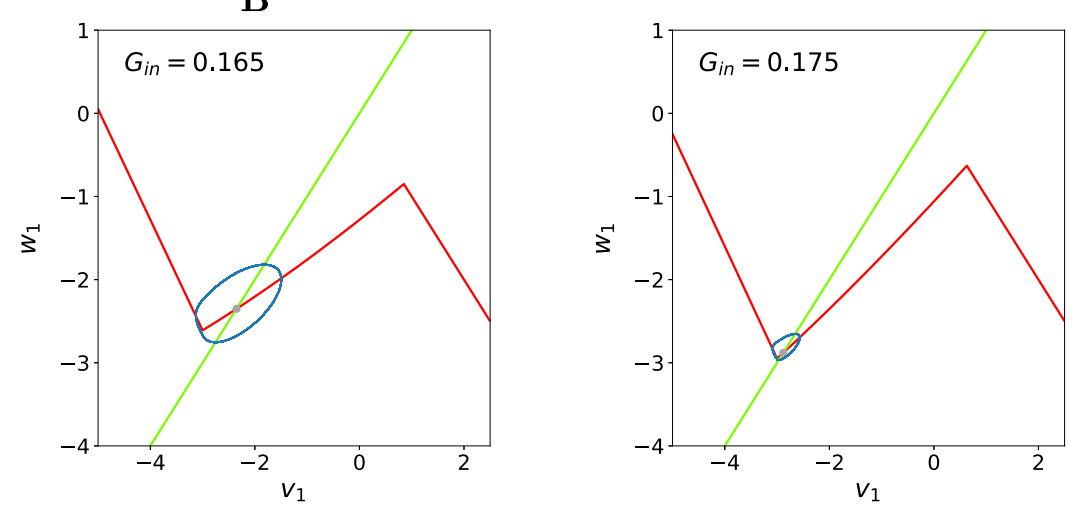

C
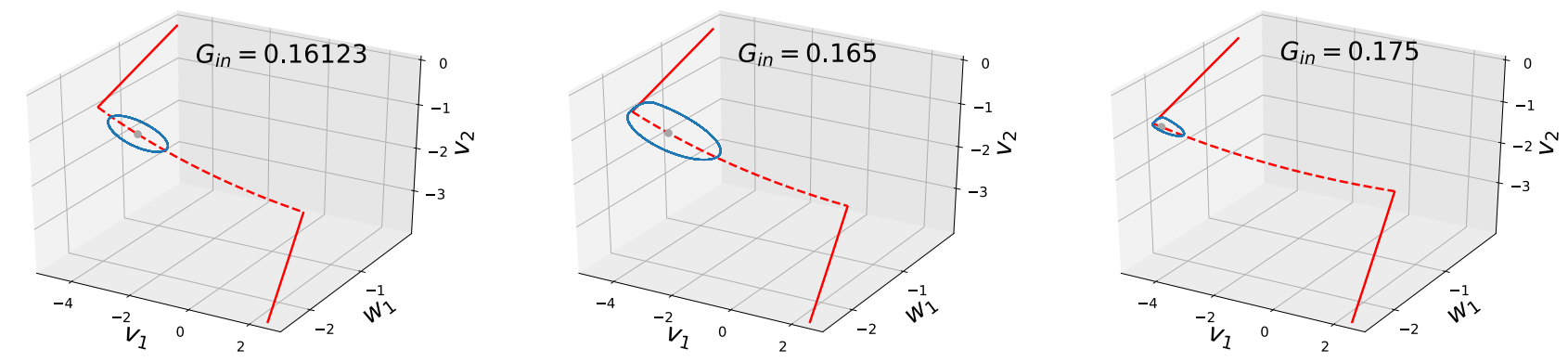

Figure 5. Non-relaxation oscillations in the PWL model for representative values of $G_{i n}$ and relatively small values of $\tau_{1}$. The values of $G_{i n}$ increase from left to right. (A) Voltage traces. (B) Cycles in the $\left(v_{1}, w_{1}\right)$-space. (C) Cycles in the $3 \mathrm{D}$ state-space. Parameter values are as in Figure 4, except for $\tau_{1}=20$.

of heteroclinic orbits connecting each fixed-point in the central branch of $C$ with the transition fixed-points $\left(-v_{a},-v_{a}, 0\right)$ or $\left(0,0,-v_{a}\right)$ (Fig. 8).

For higher values of $G_{i n}$, the fixed-point belongs to one of the attracting branches of $C$ and it becomes a stable node. Then, the transition from large amplitude oscillations to steady state solutions is abrupt when the maximal synaptic conductance is increased and the time constant $\tau_{1}$ is large enough. However, the periodic 


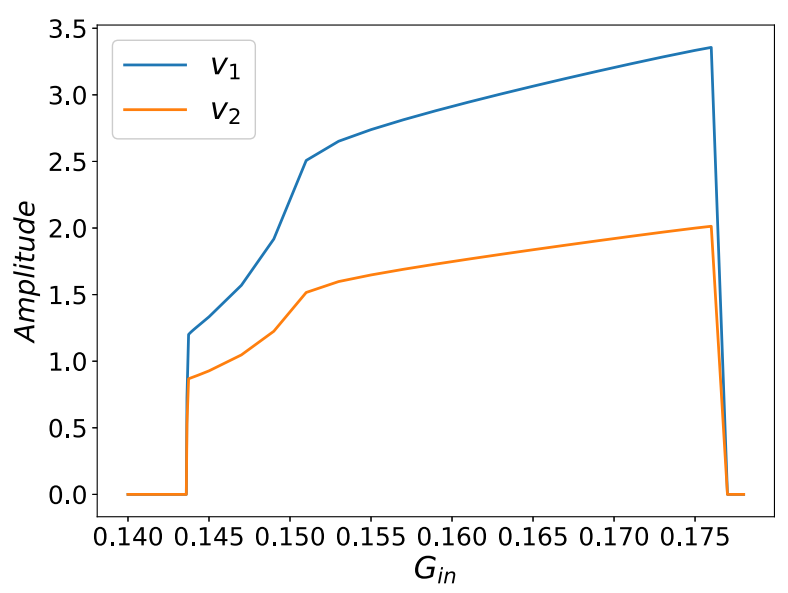

A

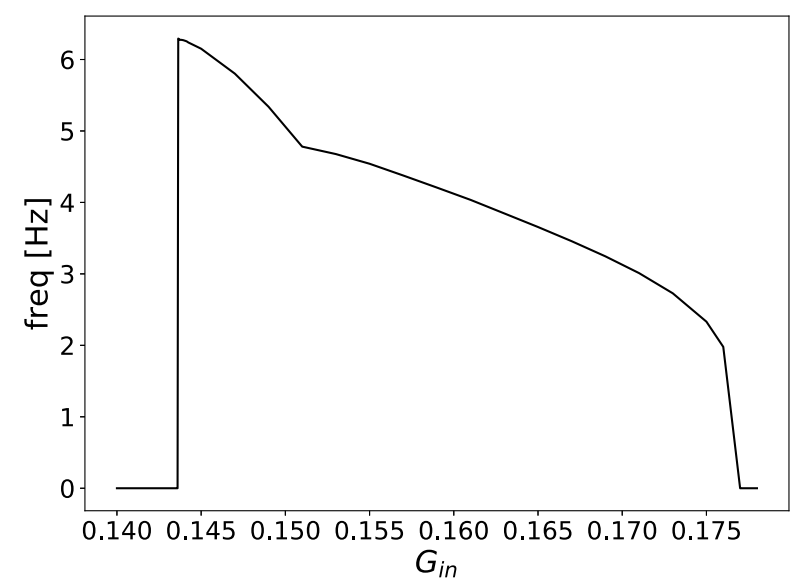

B
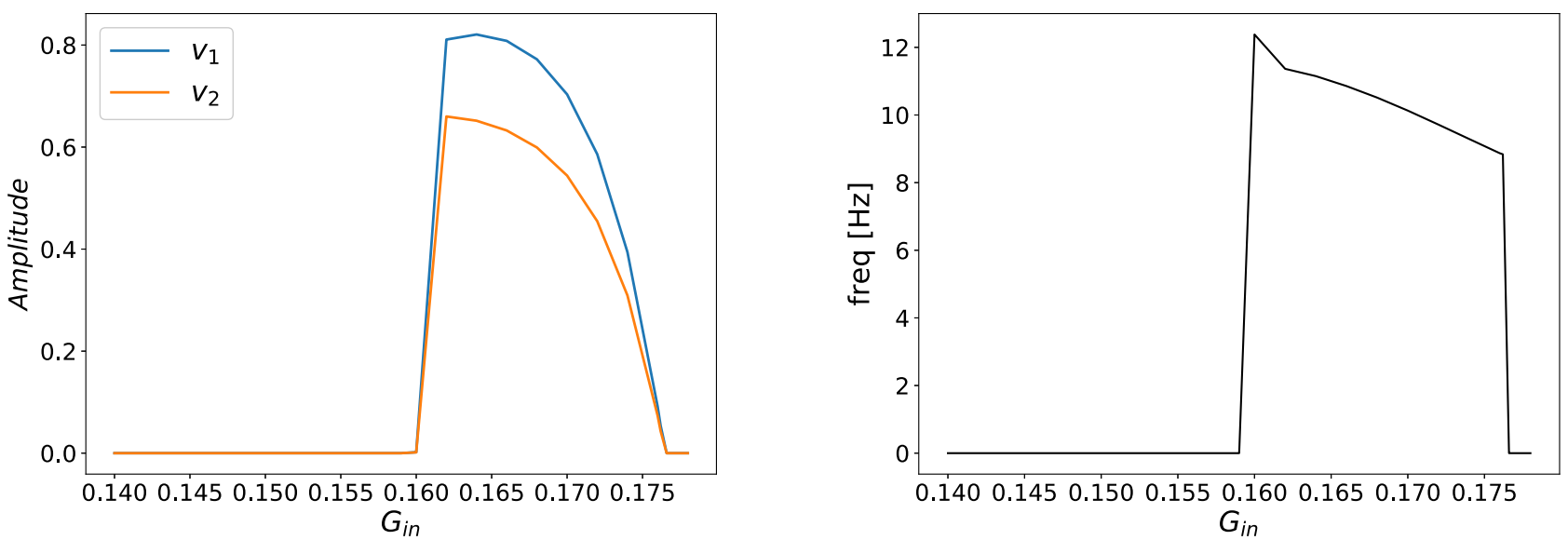

FIGURE 6. Dependence of the network oscillation amplitude and frequency with the maximal synaptic conductance $G_{i n}$. The oscillation amplitude was computed in terms of the variable $v_{1}$. (A) We use the parameter values indicated in Figure 4. (B) We use the parameter values indicated in Figure 5.

solutions do not follow the unstable branch of the critical manifold so they do not have the characteristic canard-like shape.

\subsection{A balance between $G_{i n}$ and the gradedness $v_{a}$ are necessary for network oscillations to exist in the PWL model}

The parameter $v_{a}\left(=v_{b}\right)$ in the connectivity function (2.3) determines the synapse gradedness. Increasing values of $v_{a}$ (making $S_{\infty}$ is shallower) cause the maximal synaptic conductance necessary to generate oscillation to increase (Figs. 9 and 10) while the initial frequency for small amplitude oscillations present minimal changes (not shown).

The parameter $v_{a}$ in the connectivity function (2.3) determines the synapse gradedness. We observe that increasing this value, the maximal conductance necessary to generate oscillation increases (Figs. 9 and 10) while the initial frequency for small amplitude oscillations present minimal changes (not shown). 

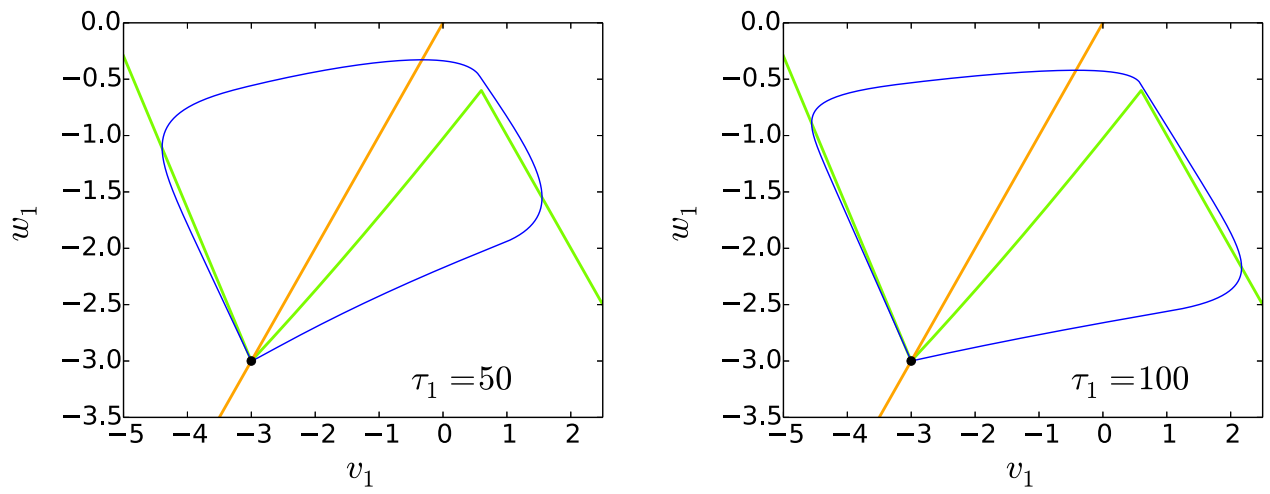

Figure 7. Homoclinic orbit varying $\tau_{1}$. We use the parameter values: $g_{L, 1}=0.25, g_{1}=0.25$, $g_{L, 2}=0.6, v_{a}=3, G_{i n}=3 / 17$ and the indicated values of $\tau_{1}$.
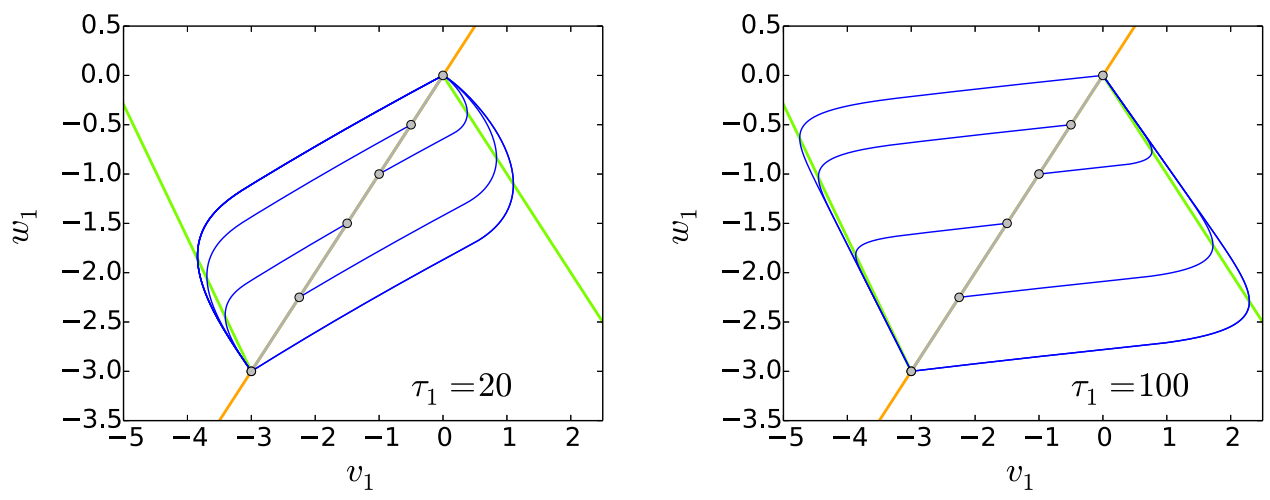

FIgURE 8 . Some heteroclinic orbits increasing $\tau_{1}$. We use the parameter values: $g_{L, 1}=0.25$, $g_{1}=0.25, g_{L, 2}=0.5, v_{a}=3, G_{i n}=3 / 17$ and the indicated values of $\tau_{1}$.

Figures 9 and 10 show the effects of increasing values of $v_{a}$ in combination with different changes in other parameter. For example, increasing values of $g_{L, 1}$ generate that critical maximal conductance $G_{i n 0}$ increases (Fig. 9A), whereas the critical parameter remains almost unchanged if $g_{L, 2}, g_{1}$ or $\tau_{1}$ are increased (Fig. 10). We calculate the critical values $G_{i n 0}$ using the frequency domain methodology (see Sect. 2.1).

The maximal conductance $G_{i n}$ and the connection gradedness $v_{a}$ has to be balanced for the existence of oscillation in the system. Suppose that the maximal conductance is fixed, $\tau_{1}$ is large enough, and we consider $v_{a}$ as bifurcation parameter. Increasing values of $v_{a}$ the attractors transition from stable fixed-points to relaxation oscillations, in such a way as we observe increasing $G_{i n}$ (Fig. 9A). For higher values of $v_{a}$ the oscillations are sinusoidal and then they disappear in an Andronov-Hopf bifurcation at a critic value of $v_{a}$ (Fig. 11). After this bifurcation the remaining attractor is the stable fixed point in the middle branch of $C$.

\subsection{The resonant frequency of the non-oscillatory resonant cell controls the network oscillation frequency}

Here we analyze how changes in resonant frequency $\left(f_{r e s}\right)$ of the non-oscillatory $2 \mathrm{D}$ cell (node 1 ) affect the network oscillation frequency $\left(f_{n t w}\right)$. For the isolated $2 \mathrm{D}$ cell, the resonant frequency and maximal impedance 

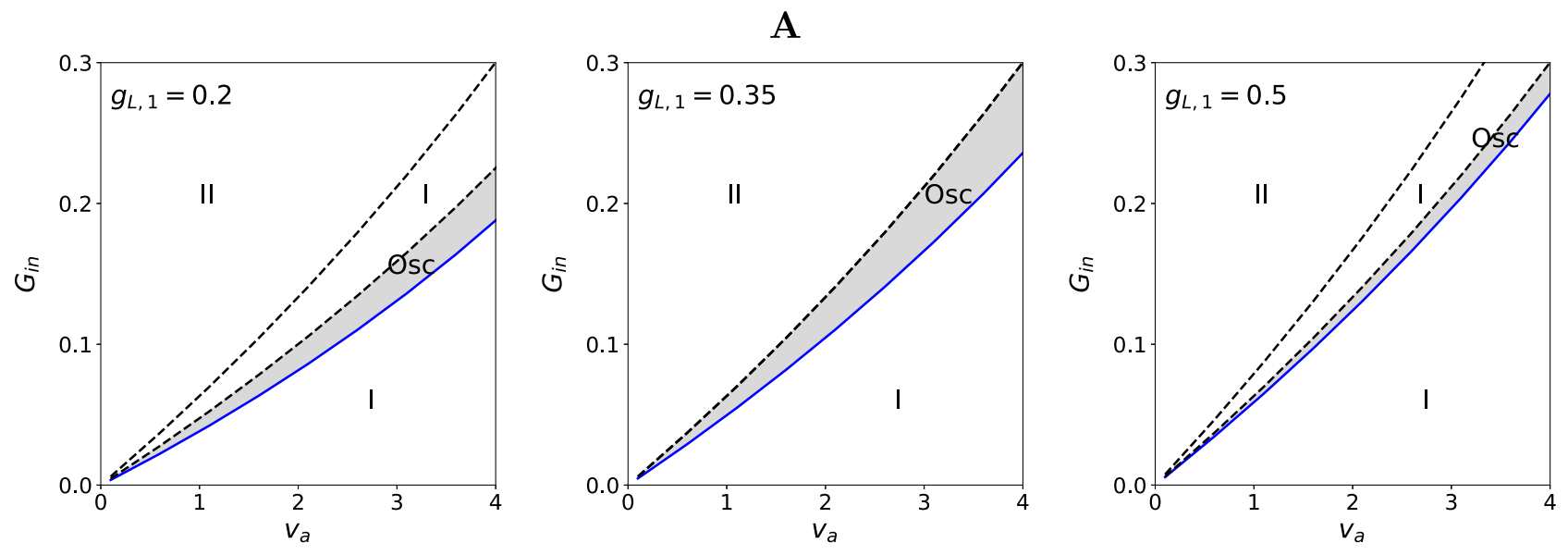

B
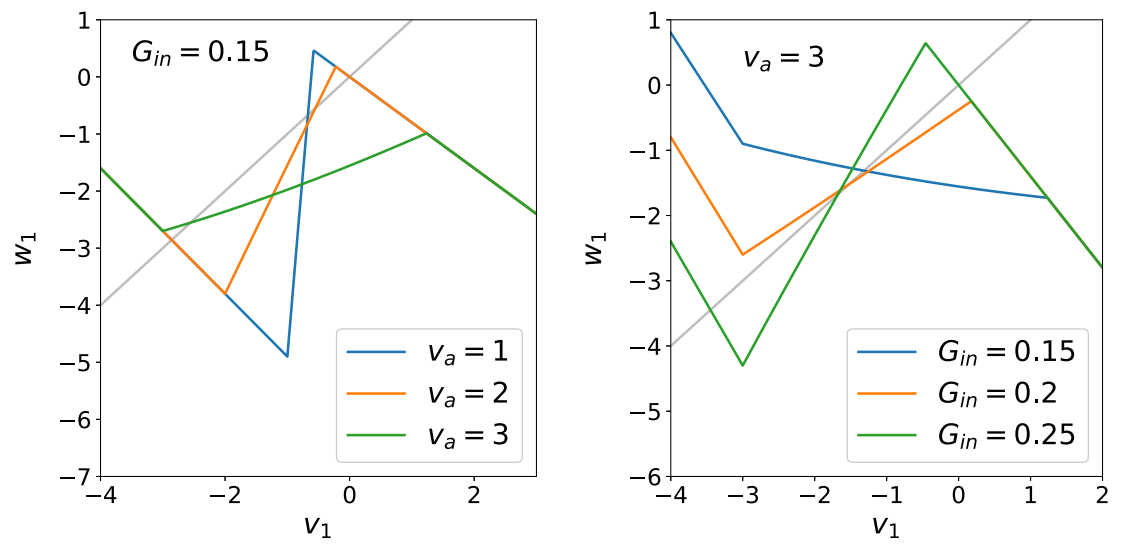

FIgURE 9. Oscillatory region and critical parameter values as function of $v_{a}$. (A) AndronovHopf bifurcation points (solid line) and values of $G_{i n, b r}$ (dashed line) as function of $v_{a}$. In the shadowed region the system presents sustained oscillations, I and II indicate the number of stable fixed-points in the corresponding region. The values of $g_{L, 1}$ are indicated in each panel. (B) Projections of $C$ and transition planes in $\left(v_{1}, w_{1}\right)$-space. Left: representative values of $v_{a}$ and fixed values of $G_{i n}=0.15$ and $g_{L, 1}=0.2$. Right: representative values of $G_{i n}$ and fixed values of $v_{a}=3$ and $g_{L, 1}=0.35$. We used the following additional parameter values: $g_{1}=0.25$, $g_{L, 2}=0.6, \tau_{1}=100$ and $E_{i n}=-20$.

are given, respectively, by

$$
f_{\text {res }}=\frac{500}{\pi} \omega_{\text {res }}=\frac{500}{\pi} \sqrt{-\frac{1}{\tau_{1}^{2}}+\frac{1}{\tau_{1}} \sqrt{g_{1}^{2}+2 g_{1} g_{L, 1}+2 \frac{g_{1}}{\tau_{1}}}}
$$

and

$$
Z_{\max }=Z\left(\omega_{\text {res }}\right)=\left(g_{L, 1}-\frac{1}{\tau_{1}}-2 \frac{g_{1}}{\tau_{1}}+\frac{2}{\tau_{1}} \sqrt{\frac{g_{1}\left(2+g_{1} \tau_{1}+2 g_{L, 1} \tau_{1}\right)}{\tau_{1}}}\right)^{-1}
$$


A

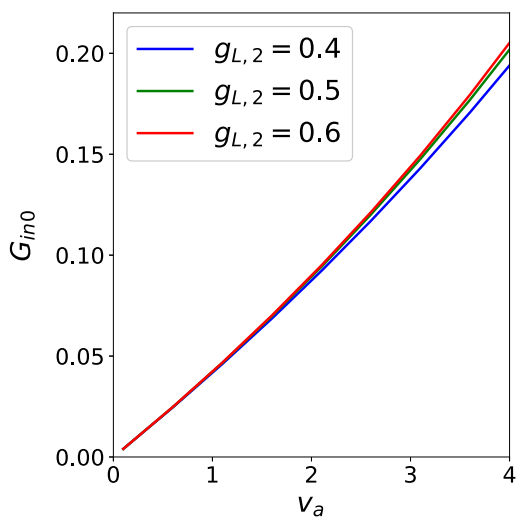

B

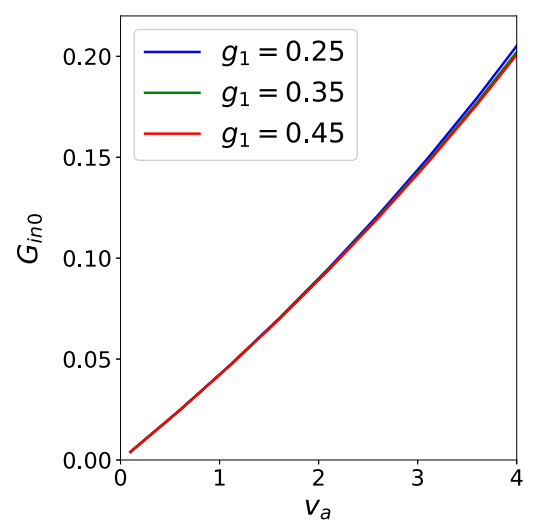

C

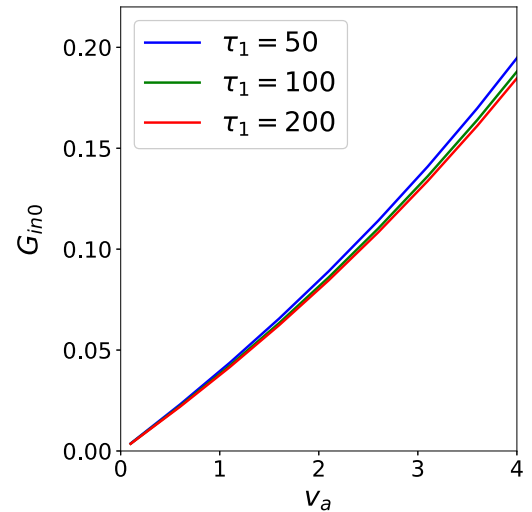

Figure 10. Critical parameter $G_{i n 0}$ as function of $v_{a}$. Andronov-Hopf critical values (solid line). (A) $g_{L, 1}=0.25, g_{1}=0.25$ and $\tau_{1}=100$. (B) $g_{L, 1}=0.25, g_{L, 2}=0.6$ and $\tau_{1}=100$. (C) $g_{L, 1}=0.2, g_{L, 2}=0.6$ and $g_{1}=0.25$.
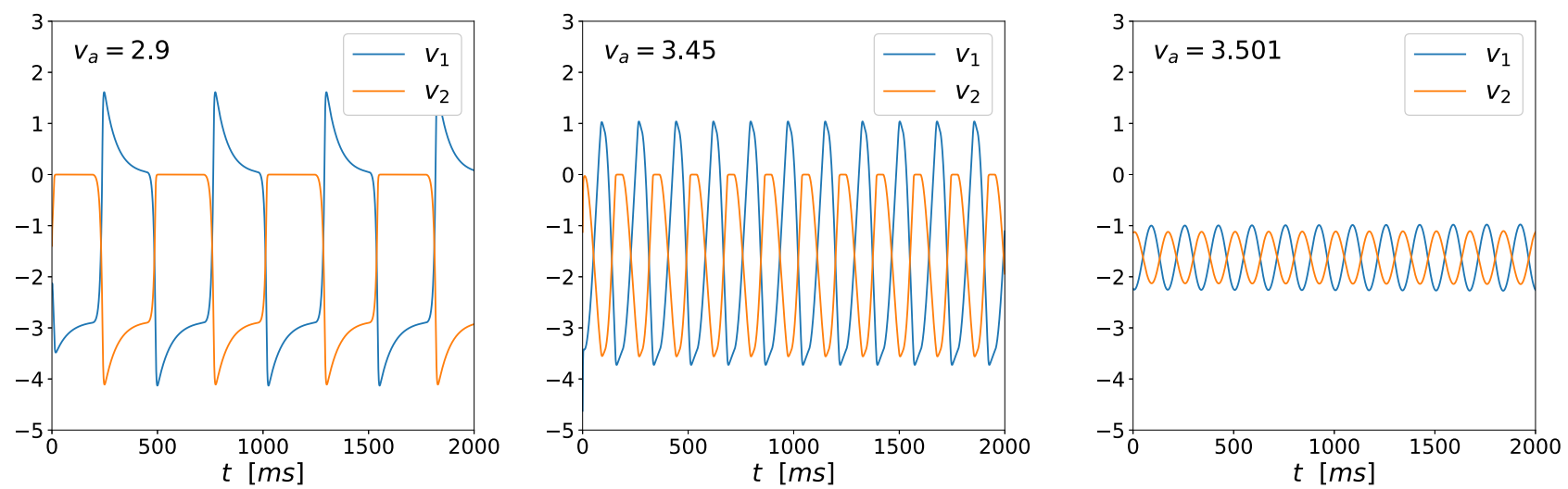

FiguRE 11. Voltage traces considering gradedness $v_{a}$ as bifurcation parameter. The values of $v_{a}$ increase from left to right. We use the following parameter values: $g_{L, 1}=0.35, g_{1}=0.25$, $g_{L, 2}=0.6, G_{i n}=0.2$ and $\tau_{1}=100$.

The factor of 500 in $f_{\text {res }}$ is used to express $f_{\text {res }}$ in $\mathrm{Hz}$.

To study the effects of $f_{\text {res }}$ on $f_{n t w}$ we follow $[4,12]$ and change the model parameters in such a way that $f_{\text {res }}$ changes but $Z_{\max }$ remains constant. In particular, we consider a fixed value of $g_{L, 1}$, and various balanced combinations of values of $g_{1}$ and $\tau_{1}$ so as to increase $f_{\text {res }}$ and maintain $Z_{\max }$ constant. In this way, the changes in $f_{\text {res }}$ maintain the impedance amplitude shape almost unchanged, and the resulting affects can be ascertained to $f_{\text {res }}$ and not the other attributes of $Z$.

In all cases considered, the network frequency $f_{n t w}$ increases with increasing values of $f_{\text {res }}$ (Figs. 12 and 13). For fixed values of $G_{i n}$, increasing the parameter $v_{a}$ we observe that the network frequency increases and the oscillation amplitude decreases (Fig. 12). As we mention in the above subsection, increasing values of the gradedness $v_{a}$ without balancing the maximal conductance $G_{i n}$ causes that oscillations to vanish, thus, we observe that the active resonant frequencies is smaller for higher values of $v_{a}$.

Figure 13 shows that the oscillations are present for higher values of $f_{\text {res }}$ and are amplified the higher $G_{i n}$. Besides, when $G_{i n}$ is increased, the oscillations present grazing bifurcations (see Sect. 3.2), and the network frequency decreases to zero when $G_{i n}$ tends to $G_{i n, b r}$. Thus, the dependence of the oscillatory frequency on 

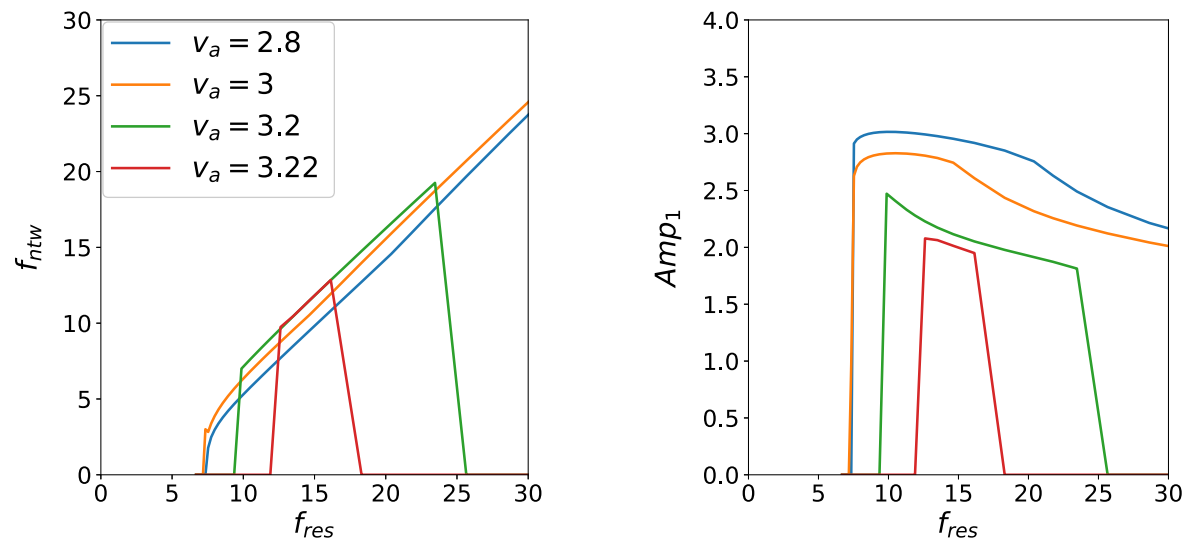

FiguRE 12. The intrinsic resonant frequency controls the network frequency. Network oscillation frequency (left) and network oscillation amplitude (right) as functions of $f_{\text {res }}$. The network oscillation amplitude was computed in terms of the amplitude of the oscillator 1 . We use the following parameter values: $g_{L, 1}=0.25, Z_{\max }=3.94$ and $G_{i n}=0.15$.

the resonant frequency is effectively lost when the oscillations become of relaxation type. This phenomenon is stronger for higher values of $g_{L, 1}$ and lower values of $Z_{\max }$.

\subsection{PWL network model without synaptic driving force}

The network model (2.1) involves the product of the PWL function of sigmoid type $S_{\infty}$ and the driving force $\left(v-E_{i n}\right)$. Here we simplify the model further by replacing this driving force factor by a positive constant (inhibition). The resulting model reads

$$
\begin{aligned}
C_{1} \frac{\mathrm{d} v_{1}}{\mathrm{~d} t} & =-g_{L, 1} v_{1}-g_{1} w_{1}-G_{i n} S_{\infty}\left(v_{2}\right) a_{1}, \\
\tau_{1} \frac{\mathrm{d} w_{1}}{\mathrm{~d} t} & =v_{1}-w_{1}, \\
C_{2} \frac{\mathrm{d} v_{2}}{\mathrm{~d} t} & =-g_{L, 2} v_{2}-G_{i n} S_{\infty}\left(v_{1}\right) a_{2},
\end{aligned}
$$

where $a_{i}>0$, for $i=1,2$.

As we discuss below, there are similarities and differences between the periodic solutions of the model (3.4) and the solutions of the model (2.1) considered in the previous subsections. The reduced model (3.4) is mathematically more tractable than the model (2.1) (it allows for analytical calculations in each linear regime) at the expense of reducing the level of biophysical descripton [13, 73, 87, 92].

As $G_{i n}$ varies, we observed that the model (3.4) presents non-smooth saddle-node bifurcations or a segment of non-isolated fixed points, depending on the values of the other parameters. This behaviour is similar to the observed for the model (2.1) (Fig. 4). However, unlike the model (2.1), because the model (3.4) is linear in each region of the state space, then Andronov-Hopf bifurcations are not possible. Despite this, the system (3.4) has periodic solutions and their expressions can be calculated by solving the linear system in each region and considering continuity conditions. The periodic solutions are generated when, by increasing the value of $G_{i n}$, the fixed point in the middle branch of the critical manifold $C$ becomes an unstable focus (Fig. 14). By necessity, the trajectories of these periodic solutions must visit at least two different regions in the three-dimensional state space, and their amplitude is determined by the position of the fixed point in the critical manifold $C$. If the value of $\tau_{1}$ is large enough, as the parameter $G_{i n}$ increases, the periodic solutions spend more time near the 
A
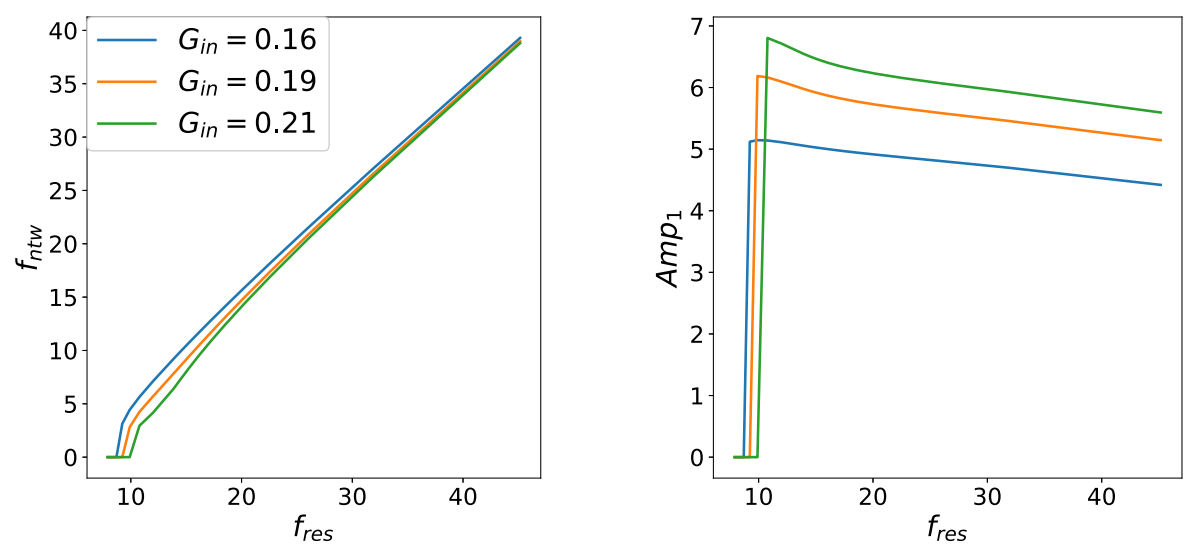

B
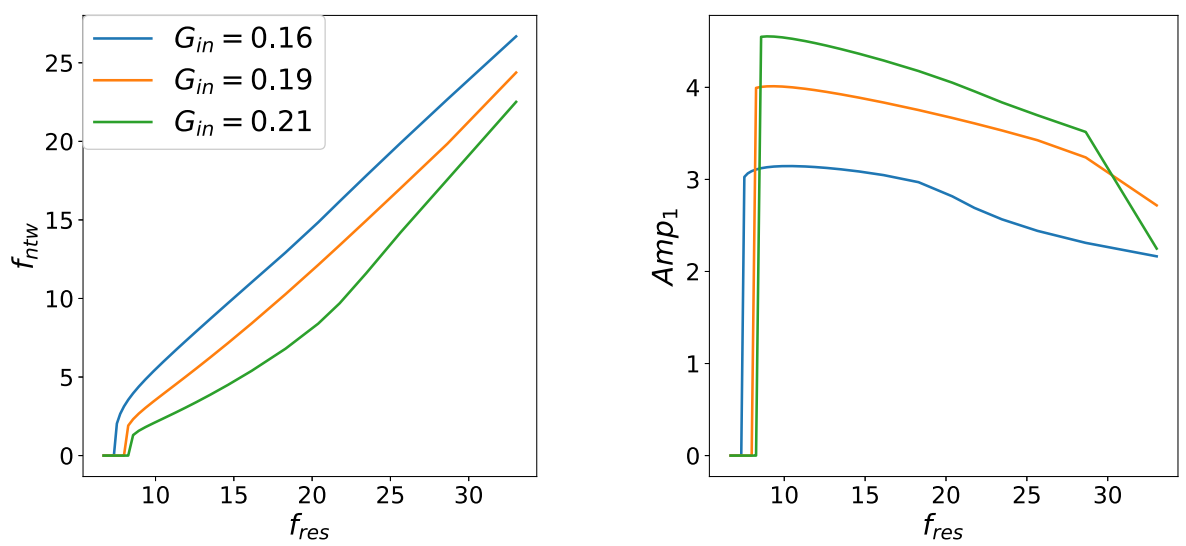

FiguRE 13. The intrinsic resonant frequency controls the network frequency. Network oscillation frequency (left) and network oscillation amplitude (oscillator 1, right) as functions of $f_{\text {res. }}$. (A) $g_{L, 1}=0.15$ and $Z_{\max }=6.4$. (B) $g_{L, 1}=0.25$ and $Z_{\max }=3.94$. We use the following parameter values $g_{L, 2}=0.6$ and $v_{a}=3$.

stable parts of $C$, and eventually take the form of relaxation oscillations (Fig. 14). When the value of $\tau_{1}$ is small enough, the fixed point is near one of the knees of the curve $C$ and small amplitude periodic solutions can be observed in (3.4) (not shown). The behaviour is similar to the one observed for the model (2.1) (Fig. 5).

\subsection{Network model with a sigmoid connectivity function revisited}

In this section, we consider the network model (2.1) with the smooth connectivity function (2.2). We refer to it simply as the sigmoid model. We studied the mechanisms that govern the emergence of oscillations in the sigmoid model in [4]. Depending on the values of the maximal conductance $G_{i n}$ the system has different number of fixed-points. Considering the maximal conductance $G_{i n}$ as bifurcation parameter, an Andronov-Hopf bifurcation around one of these fixed-points generates small amplitude sustained network oscillations (Fig. 15A). If the value of $G_{i n}$ increases (above the Andronov-Hopf bifurcation critical value) and the time constant $\tau_{1}$ is large enough, then the periodic solutions become relaxation oscillations. Thus, we conclude the effect of the two time scales present at the single cell level that give rise to resonance in the absence of intrinsic oscillations is 

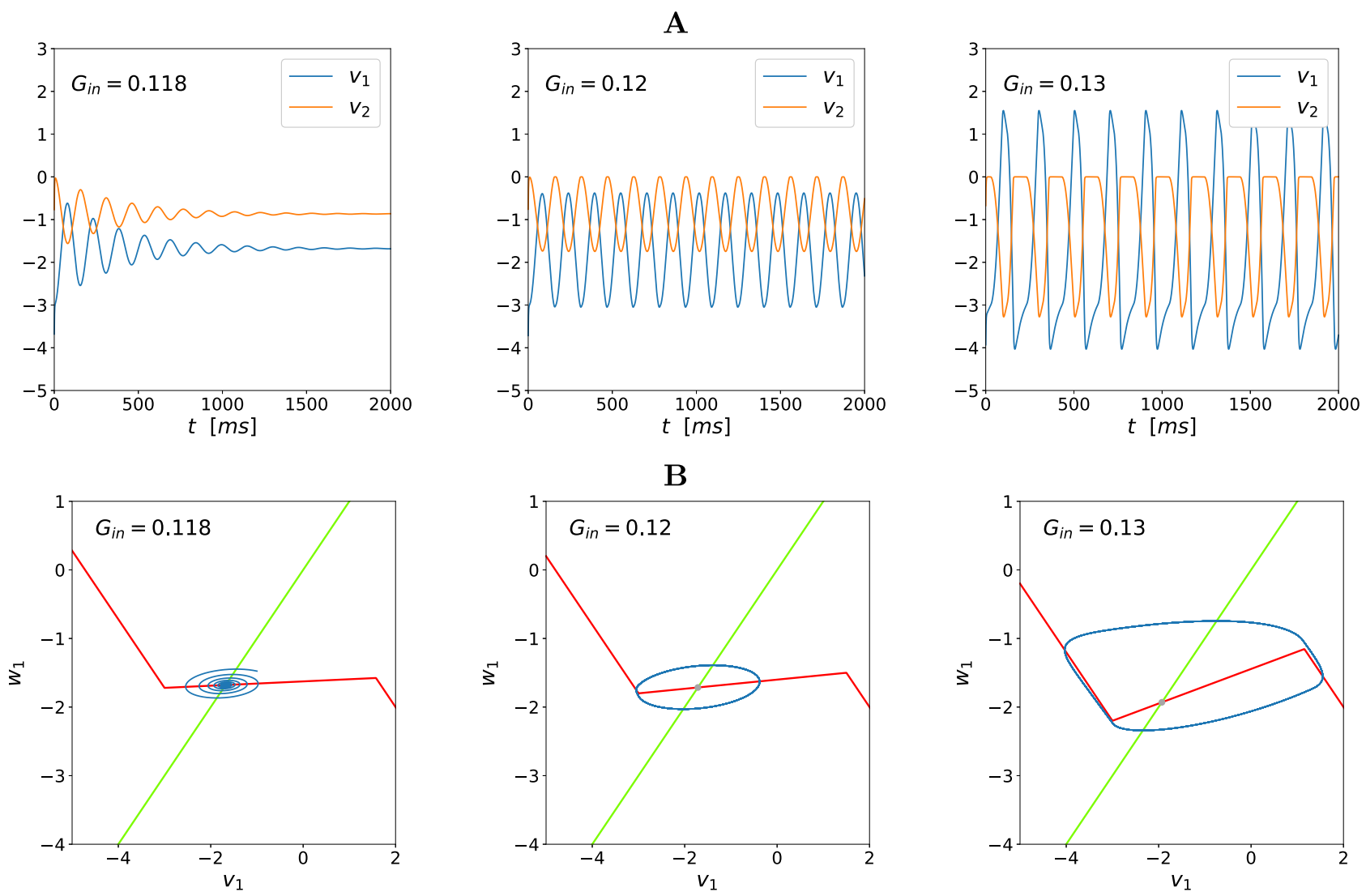

B
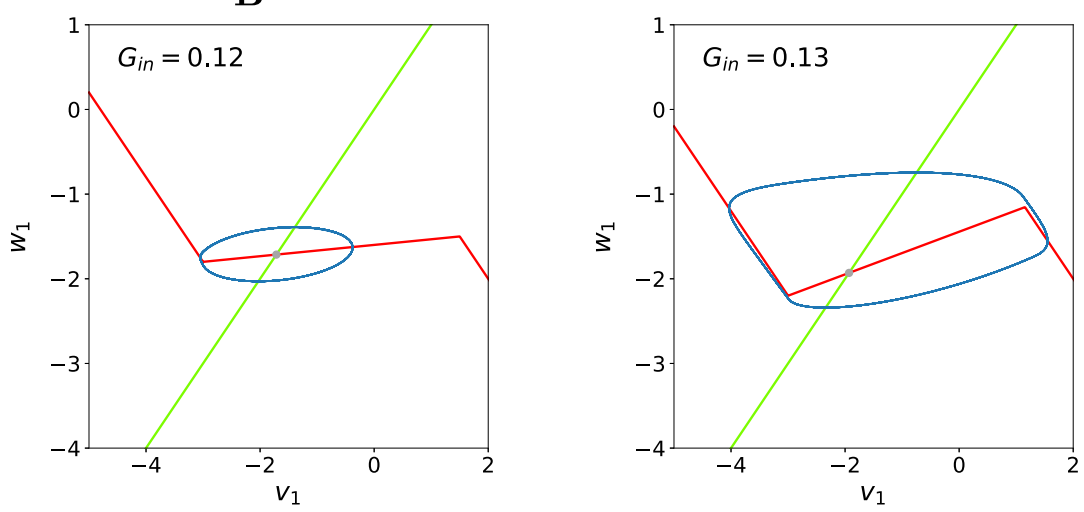

C
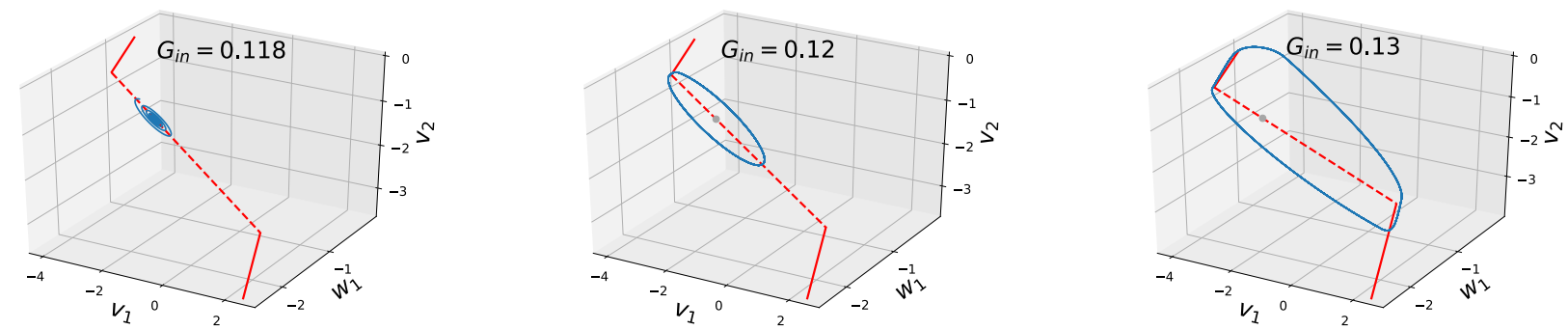

Figure 14. Generation of oscillations in the full PWL system. The values of $G_{i n}$ increase from left to right. (A) Voltage traces (curves of $v$ vs. $t$ ). (B) Trajectories in the $\left(v_{1}, w_{1}\right)$-space, and projection of the critical manifold $C$ (red PWL curve). (C) Trajectories in the 3D state-space. We used the following parameter values: $g_{L, 1}=0.25, g_{1}=0.25, g_{L, 2}=0.6, \tau_{1}=100$ and $v_{a}=3$.

translated to the network level when synaptic inhibition is strong enough. For lower levels of synaptic inhibition, the network oscillations are sinusoidal-like.

There are values of the maximal conductance $G_{i n}$ where the system presents multistability (Fig. 15B). Depending on the values of $\tau_{1}$ we observe a stable fixed-point that coexists with a stable periodic solution (Fig. 15A). This periodic solution is of relaxation type (not shown) and disappear abruptly when the maximal conductance is increased further. In the following subsection we study this behavior using a slow-fast system approach. 
A

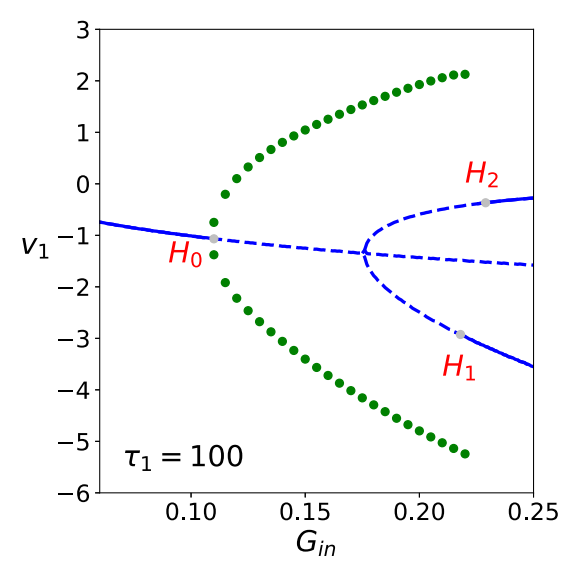

B

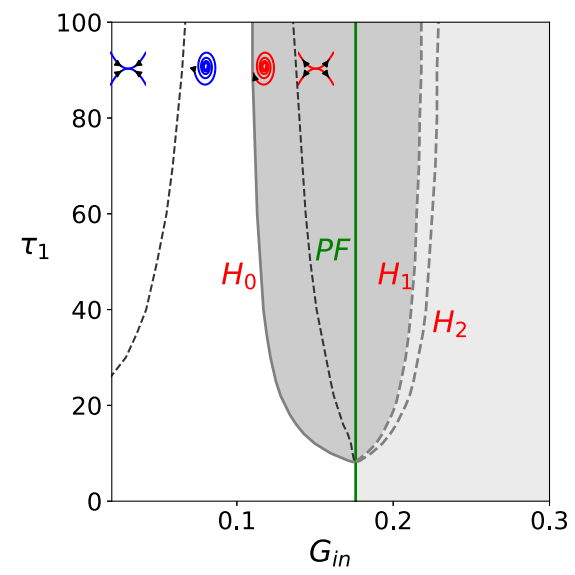

FiguRE 15. Bifurcation diagrams and region of sustained oscillations in the sigmoid model. (A) Bifurcation diagram in the $\left(G_{i n}, v_{1}\right)$ space. Stable (unstable) fixed-points in solid (dashed) line. $H_{0,1,2}$ are Andronov-Hopf critical values (gray dots). Stable sustained oscillations (green dots). (B) Bifurcation diagram in the $\left(G_{i n}, \tau_{1}\right) . H_{0,1,2}$ note the Andronov-Hopf bifurcation branches and $P F$ notes the pitchfork bifurcation. In the dark shadowed region the system presents sustained (limit cycle) oscillations. The inset trajectory diagrams indicated the dynamics within the regions bounded by the solid and dashed curves (except the solid green curve): stable nodes, stable foci, unstable foci and unstable nodes (from left to right). The inset diagrams correspond to the 3D linearized system for the fixed-point before the static bifurcation $(P F)$. The light shadowed region corresponds to multistability (limit cycle and/or fixed-points). We use the following parameter values: $g_{L, 1}=0.25, g_{1}=0.25, g_{L, 2}=0.5$.

\subsection{Canard explosion and the inflection curve method in the sigmoid model}

In a planar slow-fast dynamical system the so-called canard cycles occur near a singular Andronov-Hopf bifurcation and they enclose non-convex areas in the phase-space [6, 21, 42]. In this case an inflection point appears near the repelling slow manifold. The set of inflection points $I_{\epsilon}$ is a region in the phase-plane where the flow curvature vanishes. Therefore, the criterion for planar system states that if $\epsilon$ is small enough so that there is a set of zero curvature in the vicinity of a repelling slow manifold and a limit cycle exists, then it is possible that this cycles enclose non-convex zones, leading to a canard explosion. For a cubic shape critical manifold, the inflection set and the repelling slow manifold are $\mathcal{O}\left(\epsilon^{2}\right)$ close to one another in between both folds points of $C$.

This criterion does not establish that limit cycles exist, but only that if such cycles exist, then they can show canard solutions.

We consider the system's flow near the critical manifold $C$ (Sect. 2.2) for small values of $\epsilon=1 / \tau_{1}$. We adapt the curvature criterion mentioned before to our sigmoid model (2.1). To calculate the curvature, we consider only two components corresponding to projections onto the $\left(v_{1}, w_{1}\right)$ and $\left(v_{2}, w_{1}\right)$ planes. In the first case, we consider $v_{2}=p_{2}\left(v_{1}, G_{i n}\right)$ given in $(2.11)$, and in the second case

$$
v_{1}=p_{3}\left(v_{2}, w_{1}, G_{i n}\right)=\frac{-g_{1} w_{1}+G_{i n} S_{\infty}\left(v_{2}\right) E_{i n}}{g_{L}+G_{i n} S_{\infty}\left(v_{2}\right)} .
$$

Thus, we obtain the following two dimensional systems:

$$
\begin{array}{ll}
v_{1}^{\prime}=f_{1}\left(v_{1}, p_{2}\left(v_{1}, G_{i n}\right), w_{1}, G_{i n}\right), & v_{2}^{\prime}=f_{2}\left(p_{3}\left(v_{2}, w_{1}, G_{i n}\right), v_{2}, w_{1}, G_{i n}\right), \\
w_{1}^{\prime}=f_{3}\left(v_{1}, w_{1}\right) . & w_{1}^{\prime}=f_{3}\left(v_{1}, w_{1}\right) .
\end{array}
$$


A

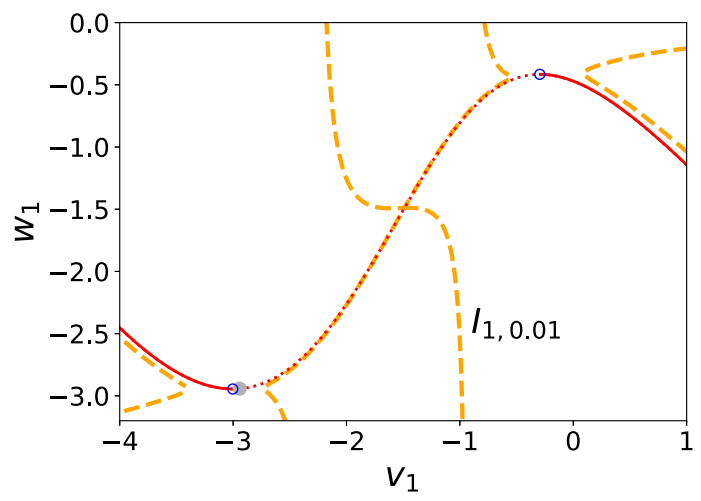

B

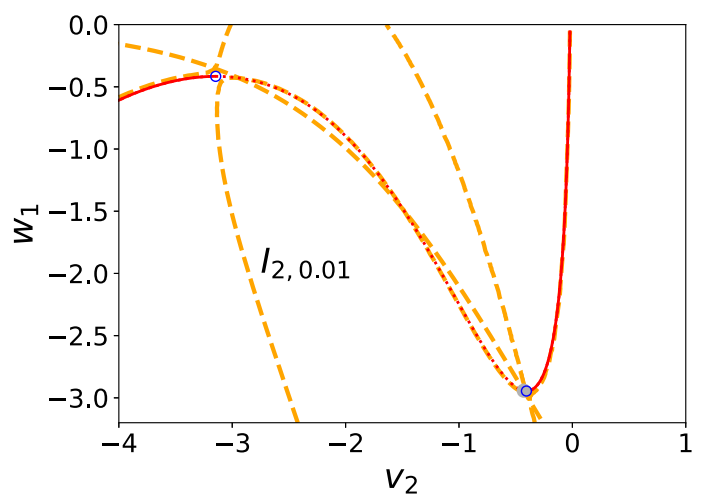

FIgURE 16. Inflection curves at the Andronov-Hopf critical value $G_{i n 0}=0.2187016$. Critical manifold $C$ (solid red) and its repelling branch (dotted red). Singular points (open blue circle) and fixed-point (filled circle). Inflection curves $I_{i, \epsilon}$ (dashed lines) for $\epsilon=0.01$. (A) Projections onto the $\left(v_{1}, w_{1}\right)$ plane. (B) Projections onto the $\left(v_{2}, w_{1}\right)$ space. We use the following parameter values: $g_{L, 1}=0.25, g_{1}=0.25$ and $g_{L, 2}=0.5$.

From these systems we define $D_{1}=\operatorname{det}\left(\begin{array}{cc}v_{1}^{\prime} & v_{1}^{\prime \prime} \\ w_{1}^{\prime} & w_{1}^{\prime \prime}\end{array}\right)$ and $D_{2}=\operatorname{det}\left(\begin{array}{cc}v_{2}^{\prime} & v_{2}^{\prime \prime} \\ w_{1}^{\prime} & w_{1}^{\prime \prime}\end{array}\right)$. The inflection points in the projections are, respectively,

$$
I_{1, \epsilon}=\left\{\left(v_{1}, w_{1}\right): D_{1}=0\right\} \quad I_{2, \epsilon}=\left\{\left(v_{2}, w_{1}\right): D_{2}=0\right\}
$$

As an example, Figure 16 shows the inflection points and the critical curve $C$ in the two projections mentioned above. We can observe that each projection of $C$ onto the corresponding planes has a cubic-like shape. The curves in Figure 16 present similarities with the inflection lines and critical manifold of the 2D Hodgkin-Huxley model studied in $[18,54]$.

Applying the methodology presented in Section 2.1, we calculate that the system presents a subcritical Andronov-Hopf bifurcation at value $G_{i n 0}=H_{1}$ (Fig. 15) and the bifurcation point is in the vicinity of the singular points (folds) of the cubic-like curves (Fig. 16). This last observation in combination with the existence of inflections points near the curve $C_{r}$ is an indicator that canard orbits can exist in the system. Performing some numerical calculations we obtain canard-like period orbits for values of the parameter $G_{i n}$ slightly greater than the Andronov-Hopf bifurcation value $H_{1}$ (Fig. 17).

It is expected that the branch of relaxation oscillations (generated in the Andronov-Hopf bifurcation at $\left.H_{0}\right)$ to fall down under the branch of unstable orbits generated in $H_{1}$, showing the typical behavior of a canard explosion. A formal proof of existence of such explosion requires the application of another methodology (analytical and numerical).

\subsection{Similarities and differences between the sigmoid (smooth) and PWL network models}

In both models, depending on the values of the parameters, the bifurcation diagrams for the fixed-points are classified into two types: the pitchfork and the saddle-node. The sigmoid model presents the classical scenario for these static bifurcations (see Fig. 15A for a pitchfork example), whereas in the PWL model the bifurcation the pitchfork bifurcation occurs through a segment of non-isolated fixed-points and the saddle-node bifurcation is non-smooth (Fig. 3). 
A
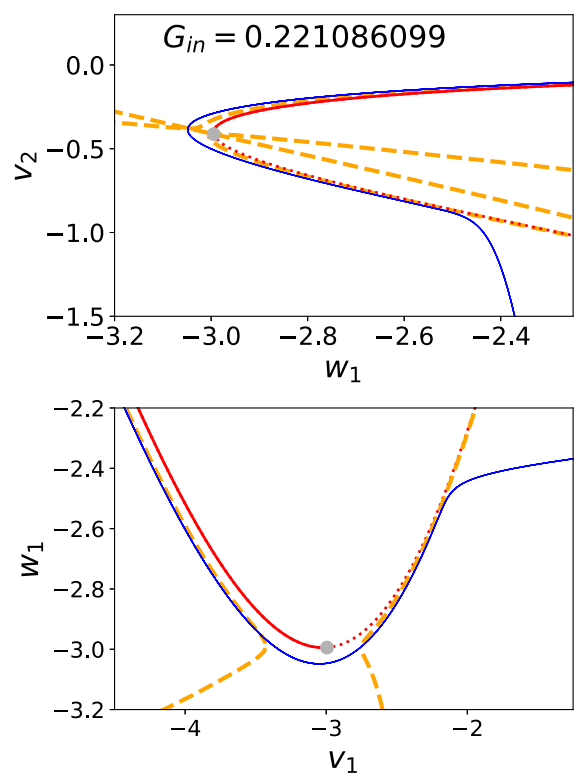

B

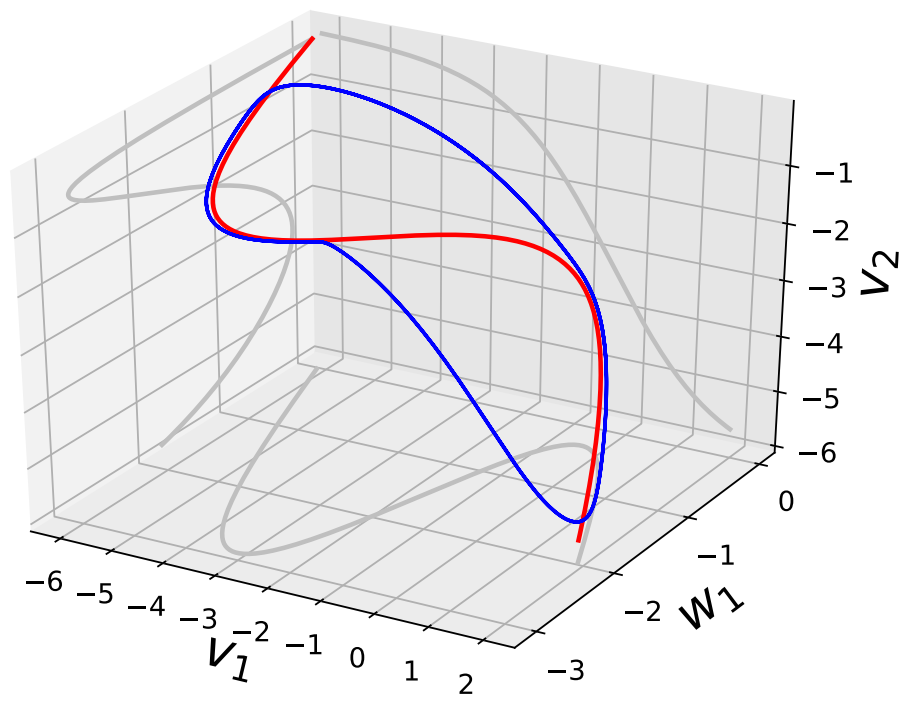

FiguRE 17. Canard-like oscillation near and Andronov-Hopf bifurcation. Value of $G_{i n}$ near the value of $G_{i n 0}$ in Figure 16. (A) Critical manifold $C$ (solid red) and its repelling branch (dotted red). Fixed-point (filled circle) and cycle (solid blue). Inflection curves $I_{i, \epsilon}$ (dashed lines) for $\epsilon=0.01$, projections onto $\left(v_{1}, w_{1}\right)$ and $\left(v_{2}, w_{1}\right)$ planes. (B) Critical manifold $C$ (red) and cycle $($ blue $)$ in 3D state-space. We use the following parameter values: $g_{L, 1}=0.25, g_{1}=0.25$, $g_{L, 2}=0.5$ and $\tau_{1}=100$.

For the two models, at critical values of the parameter $G_{i n}$ where a unique fixed-point exists, a supercritical Andronov-Hopf bifurcation appears generating a branch of stable limit cycles. This is the only dynamical bifurcation in the PWL model. In the sigmoid model two additional Andronov-Hopf bifurcations appear (both of them for greater values of $\left.G_{i n}\right)$. These last bifurcations give raise to a multistability region in the parameter space (Fig. 15).

If the constant time $\tau_{1}$ is large enough, the network presents two times scales. In both models, whenever $G_{i n}$ is increased from a critical value $G_{i n 0}$, the small amplitude oscillations (generated in an Andronov-Hopf bifurcation) evolve and become of relaxation type. However, these models present differences when the value of $G_{i n}$ increases. In the PWL model, the amplitude of the limit cycles, first grows very fast, then the cycle is transformed in a relaxation oscillation (Figs. 4 and 6A), and finally it disappears abruptly when a transition fixed-point exists (Figs. 7 and 8). In the sigmoid model, the relaxation oscillation becomes a canard-like solution, and eventually disappears abruptly when the parameter $G_{i n}$ reaches a critical value (Figs. 15 and 17).

If $\tau_{1}$ is small, both the PWL and sigmoid models present sustained oscillations which are not of relaxation type for any value of the synaptic conductance $G_{i n}$. In particular, in the PWL model, the amplitude of the oscillations is smaller than the one observed for greater values of $\tau_{1}$ (Figs. 5 and $6 \mathrm{~A}$ ) and, by increasing the values of $G_{i n}$, the cycles shrink around the same fixed-point in which they were generated. This behavior is not observed in the sigmoid model.

Finally, as we have shown in [4], in the sigmoid model the resonance frequency of the 2D node in the network controls the network frequency. This behavior is observed in the PWL model (Figs. 12 and 13) and we show that it depends on the values the gradedness of the connectivity function $v_{a}$. 


\section{Discussion}

In this paper we set out to understand the circumstances under which mutually inhibitory networks of non-oscillatory cells exhibit relaxation oscillations and the mechanisms that govern the transition from nonrelaxation, sinusoidal-like oscillations to relaxation oscillations. We used a minimal model consisting of a 2D linear resonator, a 1D linear cell and graded synaptic inhibition of PWL type. This model allows us to understand the mechanisms underlying the oscillatory phenomena in terms of a simple set of parameters for the linear components of the network.

The oscillations that emerge in this model for balanced values of $G_{i n}$ (maximal synaptic conductance) and $v_{a}$ (the synaptic gradedness) within some range are a network phenomenon since none of the components display intrinsic oscillations, consistent with our previous findings [4]. For fixed values of $v_{a}$, sinusoidal-like oscillations are created in a subcritical Hopf bifurcation, they transition to relaxation oscillations as $G_{i n}$ increases, and are abruptly terminated in a non-smooth bifurcation for higher values of $G_{i n}$ that depends on the other model parameters. The properties of the regions in the $\left(G_{i n}, v_{a}\right)$ plane for which oscillations exist depend on the parameter values for both the $1 \mathrm{D}$ cell and the $2 \mathrm{D}$ resonator. The mechanisms of generation of oscillations crucially depend on the presence of the $2 \mathrm{D}$ resonator who provides the necessary negative feedback operating at a slow time scale. Even though this feedback is not enough to produce intrinsic (damped) oscillations in the 2D node, its combination with the nonlinear amplification provided by the PWL synaptic connectivity produces oscillations at the network level.

The development of relaxation oscillations requires a relatively strong nonlinearity provided by high enough levels of $G_{i n}$ and low enough levels of $v_{a}$. In contrast to what can be expected from other systems (e.g. [48]), the relaxation oscillations are lost when the $2 \mathrm{D}$ node is a damped oscillator instead of a resonator. Damped oscillators have a smaller time scale separation between the two variables (faster negative feedback) than resonators. However, the lack of relaxation oscillations patterns is not because the trajectories become more rounded in the phase-space diagram, with an amplitude roughly of the same order of magnitude, as it would be the case for the FitzHugh-Nagumo model, for instance, but because the amplitude dependence with $G_{i n}$ is qualitatively different between the two cases. For networks having a 2D resonator, the oscillation amplitude monotonically increases with $G_{i n}$, while for networks having a 2D damped oscillator, the oscillation amplitude first increases and then decreases as $G_{i n}$ increases.

We followed previous work $[4,12]$ and investigated the dependence of the network oscillatory frequency $\left(f_{n t w}\right)$ on the properties of the resonator via its resonant frequency $\left(f_{\text {res }}\right)$, while controlling for the maximal impedance to remain fixed so as to minimize the effects that changes in $f_{\text {res }}$ have on the impedance profile. In all cases considered the $f_{n t w}$ monotonically increased with $f_{r e s}$. The range of values of $f_{\text {res }}$ for which oscillations exist critically depend on $v_{a}$, becoming narrower the larger $v_{a}$ (the shallower the synaptic connectivity function), indicating that the existence of oscillations is strengthen by nonlinearities of degree higher than two (a linear synaptic connectivity function will produce quadratic nonlinearities in the synaptic connectivity term).

Our results using the minimal networks models we considered here highlight the role that the intrinsic, nonoscillatory properties of the nodes play in the generation of network oscillations, particularly network relaxation oscillations. The mechanistic principles extracted from our study can be applied to more complex networks that can be though of as extensions of the models we considered here such as the firing rate models of Wilson-Cowan type [94] with adaptation [14, 78, 84] for neuronal populations (and networks of neuronal populations). More research is necessary to understand how the intrinsic properties of these networks affect the network oscillations.

The original Wilson-Cowan equations are an excitatory-inhibitory pair with one-dimensional dynamics for each node (population). Oscillations in this system are heavily dependent on self-connectivity in the participating nodes. In contrast, the model we present in this paper involves mutual inhibition. There are two main differences between the two formulations. First, the argument of the sigmoid function in firing rate models is the sum of all synaptic terms and the stimuli, while in our formulation the argument of the sigmoid function is the firing rate of a single node. Multiple connections in our model would involve the sum of sigmoid functions (one for each incoming input) instead of the "sigmoid of a sum". Second, the connectivity term in our model involves a driving force factor, which is absent in firing rate models. By reducing the model further such as the connectivity 
becomes piecewise liner (by replacing the diriving force of the synaptic connection by an appropriate constant), oscillations are still possible, but the mechanisms by which they are generated are different between the original and the reduced model since Andronov-Hopf bifurcations are not possible in linear systems.

A natural extension of our work is to consider larger networks involving 1D linear cells and 2D linear resonators with both inhibitory and excitatory connectivity. Future research should aim to understand how oscillations result from the interplay of the intrinsic properties of these two types of cells, the mixed connectivity and the network topology.

\section{ApPENDix A}

\section{A.1 Conductance-based models}

The linearized model (2.1) is based in biophysical (conductance-based) models [36, 79] with (at most) two ionic currents. The current balance equation to describe the neuronal subthreshold dynamics is given by

$$
C \frac{\mathrm{d} V}{\mathrm{~d} t}=-I_{L}-I_{1}-I_{2}-I_{s y n}(t)+I_{a p p}+I_{i n}(t)
$$

where $V$ is the membrane potential $(\mathrm{mV}), t$ is time $(\mathrm{ms}), C$ is the membrane capacitance $\left(\mu \mathrm{F} / \mathrm{cm}^{2}\right), I_{\text {app }}$ is the applied bias (DC) current $\left(\mu \mathrm{A} / \mathrm{cm}^{2}\right), I_{\text {in }}(t)$ is a time-dependent input current $\left(\mu \mathrm{A} / \mathrm{cm}^{2}\right), I_{L}=G_{L}\left(V-E_{L}\right)$ is the leak current, and

$$
I_{j}=G_{j} x_{j}\left(V-E_{j}\right), \quad j=1,2,
$$

are generic expressions for ionic currents with maximal conductance $G_{j}\left(\mathrm{mS} / \mathrm{cm}^{2}\right)$ and reversal potentials $E_{j}$ $(\mathrm{mV})$ respectively. The gating variables obey kinetic equations of the form

$$
\frac{\mathrm{d} x_{j}}{\mathrm{~d} t}=\frac{x_{j, \infty}(V)-x}{\tau_{j, x}(V)}
$$

where $x_{j, \infty}(V)$ and $\tau_{j, x}(V)$ are the voltage-dependent activation/inactivation curve and time constant respectively. The former is given by

$$
x_{\infty}(V)=\left(1+e^{\sigma_{x} \frac{V-V_{h l f, x}}{V_{s l p, x}}}\right)^{-1}
$$

where $V_{h l f, x}$ and $V_{s l p, x}>0$ are constants and the sign of $\sigma_{x}$ indicates whether the curve describes an activation $\left(\sigma_{x}<0\right)$ or inactivation $\left(\sigma_{x}>0\right)$ process. In all cases we use voltage-independent time constants $\tau_{j, x}$, since we are focusing on the subthreshold voltage regime where the time constants are typically slowly varying functions of $V$.

In this paper we consider 2D models with one dynamic gating variable $x_{1}$ defined by (A.3) and the other gating variable evolving on a fast time scale for which the adiabatic approximation $x_{2}=x_{2, \infty}(V)$ is made. The $1 \mathrm{D}$ models we use can be interpreted as reductions of the $2 \mathrm{D}$ models where the term including the gating variable $x_{1}$ is lacking or where the adiabatic approximations has been made for $x_{1}$.

The ionic currents we consider here are persistent sodium $I_{N a p}=G_{N a p} p_{\infty}(V)\left(V-E_{N a}\right)$, hyperpolarizationactivated, mixed-cation, inward (or h-) $I_{h}=G_{h} r\left(V-E_{h}\right)$ and slow-potassium (M-type) $I_{K s}=G_{K s} q(V-E k)$. The first one favors changes in voltage and is an example of amplifying current whereas the other two oppose changes in voltage and are called resonant currents. In the above notation we have $x_{1}=r$ and $x_{1}=q$ for the currents $I_{h}$ and $I_{K s}$, whereas $x_{2, \infty}(V)=p_{\infty}(V)$ for the current $I_{N a p}$. Functions and parameter values to define the different gating variables can be consulted, for example, in [74]. 


\section{A.2 Andronov-Hopf bifurcation in a frequency domain formulation}

In the present appendix we briefly review a formalism to study local oscillations of a dynamic system using a frequency domain approach $[52,55,89]$. By using an input-output representation, the system is described through a transfer function $\mathcal{G}(s) \in \mathbb{C}^{m \times l}$, which depends on the main (or distinguished) bifurcation parameter $\mu \in \mathbb{R}$. The aim is to determine values of $\mu$ where a classical (one limit cycle) or a degenerate (multiple limit cycles) Andronov-Hopf bifurcation arises. We apply the frequency domain approach to calculate the bifurcation points and then, with an iterative method, we obtain analytical expressions relating the bifurcation parameter and the frequency of the periodic solutions with its amplitude. These expressions allows us to describe the Andronov-Hopf bifurcation and the branch of periodic solutions generated in it.

Let $\mathcal{D}$ be an $n$-dimensional dynamical non-linear system described by

$$
\dot{x}=F(x, \mu),
$$

with $x \in \mathbb{R}^{n}, \mu \in \mathbb{R}$ is the bifurcation parameter and $F: \mathbb{R}^{n} \times \mathbb{R} \rightarrow \mathbb{R}^{n}$ is a smooth vector field $C^{k}$, with $k>3$, with equilibrium point $x=0$ satisfying $F(0, \mu)=0$.

By choosing the input and output variables appropriately, it is possible to write the following representation

$$
\mathcal{S}:\left\{\begin{array}{l}
\dot{x}=A x+B u \\
y=C x \\
u=-f(y, \mu)
\end{array}\right.
$$

which verifies that $F(x, \mu)=A x-B f(C x, \mu), A \in \mathbb{R}^{n \times n}$ is not necessarily the linearization around the equilibrium point, $B \in \mathbb{R}^{n \times l}, f: \mathbb{R}^{m} \times \mathbb{R} \rightarrow \mathbb{R}^{l}$, is $C^{k}$ with $k>3$ (it can also contain linear terms), and $C \in \mathbb{R}^{m \times n}$. The feedback is given by the function $u=-f(y, \mu), u$ is the vector of inputs and $y$ is the vector of outputs.

By applying the Laplace transform, the following representation is obtained $(\mathcal{L} y)(s)=\mathcal{G}(s)(\mathcal{L} u)(s)$, where $s$ is the Laplace variable and $\mathcal{G}(s)=C[s I-A]^{-1} B \in \mathbb{C}^{m \times l}$ is known as the transfer function.

The realization $\{A, B, C\}$ associated to $\mathcal{G}(s)$ should be minimal (controllable and observable) and, if possible, with less number of variables and equations than the original system $(m \leq n, l \leq n)$. The minimal condition assures that both representations, $\mathcal{D}$ and $\mathcal{S}$, have the same dynamical behavior, i.e. they are topological equivalent, see $[52,55,89]$.

Let $\hat{y}$ an equilibrium of $\mathcal{S}$, solution of

$$
\mathcal{G}(0) f(y, \mu)+y=0 .
$$

Then, by linearizing around $\hat{y}$, it is obtained a gain matrix $J=\left.\frac{\partial f}{\partial y}\right|_{\hat{y}}$ and an open-loop transfer function $\mathcal{G}(s) J$ for which the polynomial $\operatorname{det}(\lambda I-\mathcal{G}(s) J)=0$ defines $\lambda_{k}(s, \mu)$ characteristic functions, $k=1, \cdots, k_{0}$. Notice that $k_{0}$ is the minimum number between $m$ and $l$.

Remark A.1. The equilibrium $\hat{y}$ of $\mathcal{S}$ in the frequency domain is in correspondence with an equilibrium $\hat{x}$ of $\mathcal{D}$ in the time domain.

It is supposed that the system $\mathcal{S}$ satisfies the following conditions:

$(\mathbf{H} 1)_{\mathrm{d} f}$ : there is a simple characteristic function of the open-loop transfer matrix $\mathcal{G}(i \omega) J$, noted for simplicity as $\hat{\lambda}(\omega, \mu)$, such that for a unique frequency $\omega_{0}$ and a critical value of the parameter $\mu_{0}$ verifies $\hat{\lambda}\left(\omega_{0}, \mu_{0}\right)=-1$.

(H2) $\left.)_{\mathrm{d} f}:<\vec{\lambda}_{\partial \mu}, \vec{\lambda}_{\partial \omega}^{\perp}\right\rangle \neq 0$, where $\vec{\lambda}_{\partial \mu}=\left(\frac{\partial \Re \hat{\lambda}}{\partial \mu}\left(\omega_{0}, \mu_{0}\right), \frac{\partial \Im \hat{\lambda}}{\partial \mu}\left(\omega_{0}, \mu_{0}\right)\right), \vec{\lambda}_{\partial \omega}^{\perp}=\left(\frac{\partial \Im \hat{\lambda}}{\partial \omega}\left(\omega_{0}, \mu_{0}\right),-\frac{\partial \Re \hat{\lambda}}{\partial \omega}\left(\omega_{0}, \mu_{0}\right)\right), \Re$ and $\Im$ are the real and imaginary parts and $\langle\cdot, \cdot\rangle$ is the scalar real product.

These are classical Andronov-Hopf bifurcation conditions in the frequency domain. It is supposed here that the rest of characteristic functions do not cross the critical value -1 in the complex plane. These two conditions are the counterpart in the frequency domain to the classic conditions for the Andronov-Hopf bifurcation theorem in the time domain. 
Now, we give a version of the Andronov-Hopf bifurcation theorem using higher-order harmonic balance. Its proof, presented in [89], contains a Lyapunov Schmidt reduction method and an iterative process to determine the vectors for computing the main coefficients of the bifurcation equation up to any order. The new algorithm can be implemented simply by using symbolic computations.

Theorem A.2. Let be $\mathcal{S}$ a system that verifies $(\mathbf{H} 1)_{\mathrm{d} f}$ and $(\mathbf{H} 2)_{\mathrm{d} f}$, then the bifurcation equation of periodic orbits of higher-order in the frequency domain is

$$
\theta\left(\hat{\lambda}(\omega, \mu)+1+\sum_{k=1}^{q} \theta^{2 k} \xi_{k}(\omega, \mu)+\mathcal{O}\left(\theta^{2 q+2}\right)\right)=0 .
$$

The non-zero solutions of (A.2) are one-to-one in correspondence with the periodic solutions of small amplitude $\theta$ of the system $\mathcal{S}$ with period close to $2 \pi / \omega_{0}$.

The system $\mathcal{S}$ admits approximate oscillations of the form

$$
y(t)=\hat{y}+\sum_{j=-2 q}^{2 q} e_{j}(\omega, \mu) \exp (i j \omega t)+\mathcal{O}\left(\theta^{2 q+2}\right) .
$$

The expressions of $\xi_{k}(\omega, \mu)$ and $e_{j}(\omega, \mu)$ are obtained in the proof of the theorem.

Corollary A.3. If the conditions $(\mathbf{H} 1)_{\mathrm{d} f}$ and $(\mathbf{H 2})_{\mathrm{d} f}$ are verified, from (A.2) for $\theta \neq 0$ sufficiently small, it gives

$$
\begin{aligned}
& \mu=\mu_{0}+\mu_{2} \theta^{2}+\mu_{4} \theta^{4}+\cdots, \\
& \omega=\omega_{0}+\omega_{2} \theta^{2}+\omega_{4} \theta^{4}+\cdots .
\end{aligned}
$$

The equation (A.5) is the expression of the bifurcation of periodic solutions since it relates the main bifurcation parameter $\mu$ and the amplitude $\theta$, characterizing the Andronov-Hopf bifurcation phenomenon. The expression (A.6) gives the modification of the frequency in terms of the variation of the amplitude.

For the particular case $q=1$, if the conditions $(\mathbf{H} 1)_{\mathrm{d} f},(\mathbf{H} 2)_{\mathrm{d} f}$ and $\mu_{2} \neq 0$ are verified, the classical conditions of the Hopf bifurcation theorem in the frequency domain are satisfied and the appearance of oscillations follows the typical quadratic relation, at least locally, from the equilibrium.

For order $q>1$, an interesting question is determine which is the minimum power of $\theta$ in (A.5) that characterizes completely the local bifurcation diagrams and the types of singularities organized around a weak focus (in two dimensions) or near a degenerate Andronov-Hopf bifurcation (in three or more dimensions), i.e. a generalized Andronov-Hopf bifurcation [31, 85].

Acknowledgements. This work was partially supported by the National Science Foundation grant DMS-1608077 (H.G.R.), the NJIT Faculty Seed Grant 211278 (H.G.R.) and the Universidad Nacional del Sur grant PGI 24/L096 (A.B., A.T.). H.G.R. is grateful to the Courant Institute of Mathematical Sciences at NYU and the Department of Mathematics at Universidad Nacional del Sur, Argentina.

\section{REFERENCES}

[1] C. Ambrosio-Mouser, F. Nadim and A. Bose, The effects of varying the timing of inputs on a neural oscillator. SIAM J. Appl. Dyn. Sys. 5 (2006) 108-139.

[2] J.J. Art, A.C. Crawford and R. Fettiplace, Electrical resonance and membrane currents in turtle cochlear hair cells. Hear. Res. 22 (1986) 31-36.

[3] J. Beatty, S.C. Song and C.J. Wilson, Cell-type-specific resonances shape the response of striatal neurons to synaptic inputs. J. Neurophysiol. 113 (2015) 688-700. 
[4] A. Bel and H.G. Rotstein, Membrane potential resonance in non-oscillatory neurons interacts with synaptic connectivity to produce network oscillations. J. Comput. Neurosci. 46 (2019) 169-195.

[5] B.P. Belousov, A periodic reaction and its mechanism. Compilation of Abstracts on Radiation Medicine, Medgiz, Moscow (1959) 147-145.

[6] E. Benoit, J.L. Callot, F. Diener and M. Diener, Chasse au Canard. Collect. Math. 32 (1981) 37-76.

[7] A. Boehlen, U. Heinemann and I. Erchova, The range of intrinsic frequencies represented by medial entorhinal cortex stellate cells extends with age. J. Neurosci. 30 (2010) 4585-4589.

[8] A. Boehlen, C. Henneberger, U. Heinemann and I. Erchova, Contribution of near-threshold currents to intrinsic oscillatory activity in rat medial entorhinal cortex layer II stellate cells. J. Neurophysiol. 109 (2013) 445-463.

[9] C. Borgers, An Introduction to Modeling Neuronal Dynamics. Springer, Switzerland (2017).

[10] J.N. Brea, L.M. Kay and N.J. Kopell, Biophysical model for gamma rhythms in the olfactory bulb via subthreshold oscillations. Proc. Natl. Acad. Sci. USA 106 (2009) 21954-21959.

[11] R.L. Burden and J.D. Faires, Numerical Analysis. PWS Publishing Company, Boston (1980).

[12] Y. Chen, X. Li, H.G. Rotstein and F. Nadim, Membrane potential resonance frequency directly influences network frequency through gap junctions. J. Neurophysiol. 116 (2016) 1554-1563.

[13] S. Coombes, Neuronal networks with gap junctions: a study of piece-wise linar planar neuron models. SIAM J. Appl. Dyn. Sys. 7 (2008) 1101-1129.

[14] R. Curtu and J. Rubin, Interaction of canard and singular Hopf mechanisms in a neural model. SIAM J. Appl. Dyn. Sys. 4 (2011) 1443-1479.

[15] E. D'Angelo, S.K.E. Koekkoek, P. Lombardo, S. Solinas, E. Ros, J. Garrido, M. Schonewille and C.I. De Zeeuw, Timing in the cerebellum: oscillations and resonance in the granular layer. Neuroscience 162 (2009) 805-815.

[16] E. D'angelo, T. Nieus, A. Maffei, S. Armano, P. Rossi, V. Taglietti, A. Fontana and G. Naldi, Theta-frequency bursting and resonance in cerebellar granule cells: experimental evidence and modeling of a slow $\mathrm{K}^{+}$-dependent mechanism. J. Neurosci. 21 (2001) 759-770.

[17] F. David, E. Courtiol, N. Buonviso and N. Fourcaud-Trocme, Competing mechanisms of gamma and beta oscillations in the olfactory bulb based on multimodal inhibition of mitral cells over a respiratory cycle. eNeuro 2 (2015) e0018-15.2015.

[18] M. Desroches, M. Krupa and S. Rodrigues, Inflection, canards and excitability threshold in neuronal models. J. Math. Biol. 67 (2013) 989-1017.

[19] M. di Bernardo, C.J. Budd, A.R. Champneys and P. Kowalczyk, Piecewise-smooth Dynamical Systems. Theory and Applications. Springer-Verlag, New York (2008).

[20] M. di Bernardo, C.J. Budd, A.R. Champneys and P. Kowalczyk, Piecewise-smooth dynamical systems: theory and applications. Vol. 163 of Applied Mathematical Sciences. Springer (2008).

[21] F. Dumortier and R. Roussarie, Canard cycles and center manifolds. Memoirs of the American Mathematical Society, Rhode Island (1996) 577.

[22] T.A. Engel, L. Schimansky-Geier, A.V. Herz, S. Schreiber and I. Erchova, Subthreshold membrane-potential resonances shape spike-train patterns in the entorhinal cortex. J. Neurophysiol. 100 (2008) 1576-1588.

[23] I.R. Epstein and J.A. Pojman, An Introduction to Nonlinear Chemical Dynamics. Oxford University Press, New York (1998).

[24] I. Erchova, G. Kreck, U. Heinemann and A.V.M. Herz, Dynamics of rat entorhinal cortex layer II and III cells: Characteristics of membrane potential resonance at rest predict oscillation properties near threshold. J. Physiol. 560 (2004) 89-110.

[25] G.B. Ermentrout and D. Terman, Mathematical Foundations of Neuroscience. Springer, New York (2010).

[26] N. Fenichel, Geometric singular perturbation theory for ordinary differential equations. J. Differ. Equ. 31 (1979) 53-98.

[27] D.M. Fox, H. Tseng, T. Smolinsky, H.G. Rotstein and F. Nadim, Mechanisms of generation of membrane potential resonance in a neuron with multiple resonant ionic currents. PLoS Comput. Biol. 13 (2017) e1005565.

[28] P. Gastrein, E. Campanac, C. Gasselin, R.H. Cudmore, A. Bialowas, E. Carlier, L. Fronzaroli-Molinieres, N. Ankri and D. Debanne, The role of hyperpolarization-activated cationic current in spike-time precision and intrinsic resonance in cortical neurons in vitro. J. Physiol. 589 (2011) 3753-3773.

[29] A. Goldbeter, Biochemical Oscillations and Cellular Rhythms: The Molecular Basis of Periodic and Chaotic Behavior. Cambridge University Press, Cambridge (1996).

[30] J. Guckenheimer and P. Holmes, Nonlinear Oscillations, Dynamical Systems, and Bifurcations of Vector Fields. Springer-Verlag, New York (1983).

[31] J. Guckenheimer and P. Holmes, Nonlinear Oscillations, Dynamical Systems, and Bifurcations of Vector Fields. Vol. 42 of Applied Mathematical Sciences. Springer, Switzerland (1983).

[32] Y. Gutfreund, Y. Yarom and I. Segev, Subthreshold oscillations and resonant frequency in guinea pig cortical neurons: physiology and modeling. J. Physiol. 483 (1995) 621-640.

[33] J.G. Heys, L.M. Giacomo and M.E. Hasselmo, Cholinergic modulation of the resonance properties of stellate cells in layer II of the medial entorhinal. J. Neurophysiol. 104 (2010) 258-270.

[34] J.G. Heys, N.W. Schultheiss, C.F. Shay, Y. Tsuno and M.E. Hasselmo, Effects of acetylcholine on neuronal properties in entorhinal cortex. Front. Behav. Neurosci. 6 (2012) 32.

[35] M.H. Higgs and W.J. Spain, Conditional bursting enhances resonant firing in neocortical layer 2-3 pyramidal neurons. J. Neurosci. 29 (2009) 1285-1299.

[36] A.L. Hodgkin and A.F. Huxley, A quantitative description of membrane current and its application to conductance and excitation in nerve. J. Physiol. 117 (1952) 500-544. 
[37] H. Hu, K. Vervaeke, J.F. Storm and L.J. Graham, Complementary theta resonance filtering by two spatially segregated mechanisms in CA1 hippocampal pyramidal neurons. J. Neurosci. 29 (2009) 14472-14483.

[38] H. Hu, K. Vervaeke and J.F. Storm. Two forms of electrical resonance at theta frequencies generated by M-current, h-current and persistent $\mathrm{Na}^{+}$current in rat hippocampal pyramidal cells. J. Physiol. 545 (2002) 783-805.

[39] B. Hutcheon, R.M. Miura and E. Puil, Subthreshold membrane resonance in neocortical neurons. J. Neurophysiol. 76 (1996) 683-697.

[40] B. Hutcheon and Y. Yarom, Resonance, oscillations and the intrinsic frequency preferences in neurons. Trends Neurosci. 23 (2000) 216-222.

[41] J. Keener and J. Sneyd, Mathematical Physiology. Springer-Verlag, New York (2001).

[42] M. Krupa and P. Szmolyan, Extending geometric singular perturbation theory to nonhyperbolic points - fold and canard points in two dimensions. SIAM J. Math. Anal. 33 (2001) 286-314.

[43] C. Kuehn, Multiple Time scale Dynamics, Applied Mathematical Series. Springer-Verlag, New York (2015).

[44] I. Lampl and Y. Yarom, Subthreshold oscillations and resonant behaviour: two manifestations of the same mechanism. Neuroscience $\mathbf{7 8}$ (1997) 325-341.

[45] T. Lau and M. Zochowski, The resonance frequency shift, pattern formation, and dynamical network reorganization via sub-threshold input. PLoS ONE 6 (2011) e18983.

[46] R.R. Llinás and Y. Yarom, Oscillatory properties of guinea pig olivary neurons and their pharmachological modulation: an in vitro study. J. Physiol. 376 (1986) 163-182.

[47] Y. Manor, F. Nadim, S. Epstein, J. Ritt, E. Marder and N. Kopell, Network oscillations generated by balancing graded asymmetric reciprocal inhibition in passive neurons. J. Neurosci. 19 (1999) 2765-2779.

[48] Y. Manor, J. Rinzel, I. Segev and Y. Yarom. Low-amplitude oscillations in the inferior olive: a model based on electrical coupling of neurons with heterogeneous channel densities. J. Neurophysiol. 77 (1997) 2736-2752.

[49] B. Marcelin, A. Becker, M. Migliore, M. Esclapez and C. Bernard, h channel-dependent deficit of theta oscillation resonance and phase shift in temporal lobe epilepsy. Neurobiol. Dis. 33 (2009) 436-447.

[50] F. Marino, G. Catalán, P. Sánchez, S. Balle and O. Piro, Thermo-optical 'canard orbits' and excitable limit cycles. Phys. Rev. Lett. 92 (2004) 073901.

[51] F. Marino, F. Marin, S. Balle and O. Piro, Chaotically spiking canards in an excitable system with 2D inertial fast manifolds. Phys. Rev. Lett. 98 (2007) 074104.

[52] A.I. Mees, Dynamics of Feedback Systems. John Wiley \& Sons, Chichester, UK (1981).

[53] J. Mikiel-Hunter, V. Kotak and J. Rinzel, High-frequency resonance in the gerbil medial superior olive. PLoS Comput. Biol. 12 (2016) 1005166.

[54] J. Moehlis, Canard for a reduction of the Hodgkin-Huxley equations. J. Math. Biol. 52 (2006) 141-153.

[55] J.L. Moiola and G. Chen, Hopf Bifurcation Analysis: A Frequency Domain Approach, Vol. 21 of World Scientific Series on Nonlinear Science. World Scientific Publishing, Singapore (1996).

[56] R. Muresan and C. Savin, Resonance or integration? Self-sustained dynamics and excitability of neural microcircuits. J. Neurophysiol. 97 (2007) 1911-1930.

[57] J.D. Murray, Mathematical Biology: I. An Introduction. Springer, Berlin (2002).

[58] R. Narayanan and D. Johnston, Long-term potentiation in rat hippocampal neurons is accompanied by spatially widespread changes in intrinsic oscillatory dynamics and excitability. Neuron 56 (2007) 1061-1075.

[59] R. Narayanan and D. Johnston, The h channel mediates location dependence and plasticity of intrinsic phase response in rat hippocampal neurons. J. Neurosci. 28 (2008) 5846-5850.

[60] M.F. Nolan, J.T. Dudman, P.D. Dodson and B. Santoro, HCN1 channels control resting and active integrative properties of stellate cells from layer II of the entorhinal cortex. J. Neurosci. 27 (2007) 12440-12551.

[61] F.G. Pike, R.S. Goddard, J.M. Suckling, P. Ganter, N. Kasthuri and O. Paulsen, Distinct frequency preferences of different types of rat hippocampal neurons in response to oscillatory input currents. J. Physiol. 529 (2000) 205-213.

[62] R.K. Rathour and R. Narayanan, Inactivating ion channels augment robustness of subthreshold intrinsic response dynamics to parametric variability in hippocampal model neurons. J. Physiol. 590 (2012) 5629-5652.

[63] R.K. Rathour and R. Narayanan, Homeostasis of functional maps in inactive dendrites emerges in the absence of individual channelostasis. Proc. Natl. Acad. Sci. USA 111 (2014) E1787-E1796.

[64] F. Rau, J. Clemens, V. Naumov, R.M. Hennig and S. Schreiber, Firing-rate resonances in the peripheral auditory system of the cricket, gryllus bimaculatus. J. Comp. Physiol. 201 (2015) 1075-1090.

[65] W.H. Remme, R. Donato, J. Mikiel-Hunter, J.A. Ballestero, S. Foster, J. Rinzel and D. McAlpine, Subthreshold resonance properties contribute to the efficient coding of auditory spatial cues. Proc. Natl. Acad. Sci. USA 111 (2014) E2339-E2348.

[66] M.J.E. Richardson, N. Brunel and V. Hakim, From subthreshold to firing-rate resonance. J. Neurophysiol. 89 (2003) $2538-2554$.

[67] B. Romeira, J. Javaloyes, S. Balle, O. Piro, R. Avó and J. Figueiredo, Neuromorphic opto-electronic integrated circuits for optical signal processing. Proc. SPIE- Int. Soc. Opt. Eng. 9286 (2014) 928606.

[68] B. Romeira, J. Javaloyes, C.N. Ironside, M.L. José Figueiredo, S. Balle and O. Piro, Excitability and optical pulse generation in semiconductor lasers driven by resonant tunneling diode photo-detectors. Opt. Express 21 (2013) 20931-20940.

[69] H.G. Rotstein, Frequency preference response to oscillatory inputs in two-dimensional neural models: a geometric approach to subthreshold amplitude and phase resonance. J. Math. Neurosci. 4 (2014) 11.

[70] H.G. Rotstein, Subthreshold amplitude and phase resonance in models of quadratic type: nonlinear effects generated by the interplay of resonant and amplifying currents. J. Comp. Neurosci. 38 (2015) 325-354. 
[71] H.G. Rotstein, Resonance modulation, annihilation and generation of antiresonance and antiphasonance in $3 \mathrm{~d}$ neuronal systems: interplay of resonant and amplifying currents with slow dynamics. J. Comp. Neurosci. 43 (2017) 35-63.

[72] H.G. Rotstein, The shaping of intrinsic membrane potential oscillations: positive/negative feedback, ionic resonance/ amplification, nonlinearities and time scales. J. Comp. Neurosci. 42 (2017) 133-166.

[73] H.G. Rotstein, S. Coombes and A.M. Gheorghe, Canard-like explosion of limit cycles in two-dimensional piecewise-linear models of FitzHugh-Nagumo type. SIAM J. Appl. Dyn. Syst. 11 (2012) 135-180.

[74] H.G. Rotstein and F. Nadim, Frequency preference in two-dimensional neural models: a linear analysis of the interaction between resonant and amplifying currents. J. Comp. Neurosci. 37 (2014) 9-28.

[75] F. Sagués and I.R. Epstein, Nonlinear chemical dynamics. Dalton Trans. 3 (2003) 1201-1217.

[76] S. Schreiber, I Erchova, U. Heinemann and A.V. Herz, Subthreshold resonance explains the frequency-dependent integration of periodic as well as random stimuli in the entorhinal cortex. J. Neurophysiol. 92 (2004) 408-415.

[77] G. Sciamanna and C.J. Wilson, The ionic mechanism of gamma resonance in rat striatal fast-spiking neurons. J. Neurophysiol. 106 (2011) 2936-2949.

[78] A. Shpiro, R. Moreno-Bote, N. Rubin and J. Rinzel, Balance between noise and adaptation in competition models of perceptual bistability. J. Comp. Neurosci. 27 (2009) 37-54.

[79] F.K. Skinner, Conductance-based models. Scholarpedia 1 (2006) 1408.

[80] S. Solinas, L. Forti, E. Cesana, J. Mapelli, E. De Schutter and E. D'Angelo, Fast-reset of pacemaking and theta-frequency resonance in cerebellar Golgi cells: simulations of their impact in vivo. Front. Cell. Neurosci. 1 (2007) 4.

[81] S.C. Song, J.A. Beatty and C.J. Wilson, The ionic mechanism of membrane potential oscillations and membrane resonance in striatal lts interneurons. J. Neurophysiol. 116 (2016) 1752-1764.

[82] S.H. Strogatz, Nonlinear Dynamics and Chaos. Addison Wesley, Reading MA (1994).

[83] A. Szucs, A. Ráktak, K. Schlett and R. Huerta, Frequency-dependent regulation of intrinsic excitability by voltage-activated membrane conductances, computational modeling and dynamic clamp. Eur. J. Neurosci. 46 (2017) 2429-2444.

[84] J. Tabak, J. Rinzel and R. Bertram, Quantifying the relative contributions of divisive and substractive feedback to rhythm generation. PLoS Comput. Biol. 7 (2011) e1001124.

[85] F. Takens, Unfoldings of certain singularities of vectorfields: Generalized hopf bifurcations. J. Differ. Equ. 14 (1973) $476-493$.

[86] V. Tohidi and F. Nadim, Membrane resonance in bursting pacemaker neurons of an oscillatory network is correlated with network frequency. J. Neurosci. 29 (2009) 6427-6435.

[87] A. Tonnelier, The McKean's caricature of the FitzHugh-Nagumo model. The space-clamped system. SIAM J. Appl. Math. 63 (2002) 459-484.

[88] B. Torben-Nielsen, I. Segev and Y. Yarom, The generation of phase differences and frequency changes in a network model of inferior olive subthreshold oscillations. PLoS Comput. Biol. 8 (2012) 31002580.

[89] A.M. Torresi, G.L. Calandrini, P.A. Bonfili and J.L. Moiola, Generalized Hopf bifurcation in a frequency domain formulation. Int. J. Bifurc. Chaos 22 (2012) 1-16.

[90] H. Tseng and F. Nadim, The membrane potential waveform on bursting pacemaker neurons is a predictor of their preferred frequency and the network cycle frequency. J. Neurosci. 30 (2010) 10809-10819.

[91] J.F.M. van Brederode and A.J. Berger, Spike-firing resonance in hypoglossal motoneurons. J. Neurophysiol. 99 (2008) 29162928.

[92] W.P. Wang, Multiple impulse solutions to McKean's caricature of the nerve equation. 1. Existence. Commun. Pure Appl. Math. 41 (1988) 71-103.

[93] X.-J. Wang and J. Rinzel, Alternating and synchronous rhythms in reciprocally inhibitory model neurons. Neural Comput. 4 (1992) 84-97.

[94] H.R. Wilson and J.D. Cowan, Excitatory and inhibitory interactions in localized populations of model neurons. Biophys. J. 12 (1972) 1-24.

[95] A.T. Winfree, The Geometry of Biological Time, 2nd edn. Springer-Verlag, New York (2001).

[96] N. Wu, C.-F. Hsiao and S.H. Chandler, Membrane resonance and subthreshold membrane oscillations in mesencephalic V neurons: participants in burst generation. J. Neurosci. 21 (2001) 3729-3739.

[97] S. Yang, W. Lin and A.A. Feng, Wide-ranging frequency preferences of auditory midbrain neurons: roles of membrane time constant and synaptic properties. Eur. J. Neurosci. 30 (2009) 76-90.

[98] R. Zemankovics, S. Káli, O. Paulsen, T.F. Freund and N. Hájos, Differences in subthershold resonance of hippocampal pyramidal cells and interneurons: the role of h-current and passive membrane characteristics. J. Physiol. 588 (2010) $2109-2132$.

[99] A.M. Zhabotinsky, Periodic processes of malonic acid oxidation in a liquid phase. Biofizika 9 (1964) $306-311$. 